\title{
Un enfoque regional para estudiar la diversidad industrial en la Argentina (1996-2012) ${ }^{1}$
}

\author{
Andrea Belmartino y Carla Daniela Calá
}

\section{Resumen}

El objetivo de este artículo es cuantificar la diversidad productiva de la industria manufacturera en las provincias argentinas, analizar su evolución entre 1996 y 2012, e identificar los principales factores económicos asociados a este fenómeno. Se calcula un índice de diversidad a partir de datos oficiales del total de empleo asalariado registrado, provistos por la Base de Datos para el Análisis Dinámico del Empleo. Luego se analiza la evolución de la diversidad durante el período en las distintas provincias y finalmente se estima un modelo econométrico de datos de panel para identificar los principales factores económicos asociados. La diversidad industrial de las provincias se asocia negativamente con la salida de empresas y positivamente con el grado de desarrollo, el tamaño de la región, una mayor urbanización y mayores capacidades territoriales. Estos resultados pueden servir para el diseño de políticas que fomenten la diversidad regional.

\section{Palabras clave}

Industria, empresas industriales, empresas manufactureras, productos manufacturados, modelos econométricos, estadísticas industriales, Argentina

\section{Clasificación JEL}

$$
\text { L60, R11 }
$$

\section{Autores}

Andrea Belmartino es ayudante graduada de dedicación exclusiva en el Centro de Investigaciones Económicas de la Facultad de Ciencias Económicas y Sociales de la Universidad Nacional de Mar del Plata, Argentina. Correo electrónico: belmartino@mdp.edu.ar.

Daniela Calá es profesora adjunta de dedicación exclusiva en el Centro de Investigaciones Económicas de la Facultad de Ciencias Económicas y Sociales de la Universidad Nacional de Mar del Plata, Argentina. Correo electrónico: dacala@mdp.edu.ar.

Las autoras agradecen al Observatorio de Empleo y Dinámica Empresarial (Ministerio de Trabajo, Empleo y Seguridad Social de la Nación) por facilitar el acceso a los datos, a Javier Alejo, de la Universidad Nacional de La Plata (UNLP), y a los participantes de la XXI Reunión Anual de la Red Pymes MERCOSUR (Tandil, 2016) y de la VI Reunión Anual de la Asociación Argentina de Economía Política (Tucumán, 2016) por sus valiosos comentarios a versiones anteriores a este trabajo. 


\section{Introducción}

La diversidad productiva es un elemento clave en el diseño de estrategias de desarrollo de los países. En particular, la variedad de bienes producidos y exportados está directamente relacionada con el desarrollo económico en sus etapas iniciales (Imbs y Wacziarg, 2003; Klinger y Lederman, 2004; Hidalgo y Hausmann, 2010). A su vez, la diversidad de estructuras productivas genera beneficios adicionales, como el fomento de la innovación y la transmisión del conocimiento (Duranton y Puga, 2000; Frenken, Van Oort y Verburg, 2007), la reducción de la vulnerabilidad a perturbaciones externas (Ghosh y Ostry, 1994; Kosacoff y Ramos, 1999; Haddad, Lim y Saborowski, 2010), incentivos para el ingreso de nuevas empresas (Guesnier, 1994; Reynolds, Storey y Westhead, 1994) y mayores oportunidades de inversión (Al-Marhubi, 2000; Herzer y Nowak-Lehnmann, 2006; Hesse, 2009).

Estos hallazgos empíricos otorgan a la diversidad productiva un papel central en el diseño de políticas industriales de desarrollo local. Por ende, resulta importante conocer cuán diversas son las estructuras productivas regionales, cómo ha evolucionado esa diversidad a lo largo del tiempo y cuáles son los factores económicos asociados. Este artículo aporta una mirada regional sobre este fenómeno a partir de un análisis doble: por un lado, se describe la evolución de la diversidad productiva de la industria en las provincias argentinas entre 1996 y 2012, y por otro, se examina la relación entre la diversidad industrial y algunas características de las regiones, tales como su grado de desarrollo, nivel de urbanización, existencia de recursos y capacidades y variables de dinámica empresarial. A partir de allí se busca generar información relevante que pueda ser utilizada en el diseño de políticas de desarrollo industrial a escala regional.

Los resultados obtenidos permiten avanzar en la comprensión de la diversidad productiva, que la Comisión Económica para América Latina y el Caribe (CEPAL) ha considerado un mecanismo relevante para desarrollar nuevas capacidades tecnológicas en el marco de la implementación de políticas industriales selectivas (Lavarello y Sarabia, 2015). A ese respecto, la CEPAL -en línea con la Agenda 2030 para el Desarrollo Sostenible-, planteó en 2017 la necesidad de transitar hacia sectores más intensivos en conocimiento y que faciliten la diversificación productiva, para así generar nuevas oportunidades de integración e inclusión social².

En la Argentina este tema también ha sido destacado en los lineamientos del Plan Estratégico Industrial 2020 (Ministerio de Industria, 2011), donde se señala la importancia de generar acciones que promuevan una trama productiva más diversa a fin de lograr un crecimiento sostenible y con equidad (Porta, 2016). Sin embargo, este creciente interés se contrapone con una escasa cantidad de contribuciones académicas que aborden empíricamente el fenómeno desde una perspectiva regional. Los trabajos que estudian la diversidad productiva en la Argentina se enfocan fundamentalmente en el análisis de las exportaciones a nivel país (Bebczuk y Berrettoni, 2006), o en la distribución del empleo de determinadas áreas geográficas (Fritzsche y Vio, 2000; Rojo Brizuela y Rotondo, 2006; Mazorra y Beccaria, 2007).

El artículo se estructura de la siguiente forma: en primer lugar, se resumen los principales aportes teóricos y empíricos que permiten identificar y comprender los factores económicos asociados a la diversidad productiva regional. Luego se describen la fuente de datos, el índice de diversidad utilizado y la estrategia de estimación. A continuación, se enumeran los resultados y, por último, se presentan algunas reflexiones y posibles líneas futuras de investigación.

\footnotetext{
2 El trabajo de Bielschowsky, Izam y Mulder (2011) presenta una síntesis de la evolución del pensamiento de la CEPAL sobre la diversificación productiva y la inserción internacional desde 1950 hasta la actualidad.
} 


\section{Marco teórico}

Este trabajo se enmarca en el campo de la ciencia regional, es decir, en el conjunto de estudios que incorporan la dimensión territorial y sus heterogeneidades en el análisis económico (Moncayo Jiménez, 2001). Si bien este campo de la ciencia ha proporcionado modelos teóricos que permiten el estudio riguroso de ciertos temas como el crecimiento regional o la concentración territorial de la actividad económica (Capello, 2006), aún no existen modelos que describan adecuadamente el fenómeno de la diversidad productiva regional. Es por eso que los estudios empíricos sobre el tema (véase la sección III) suelen utilizar especificaciones econométricas ad hoc, incorporando elementos procedentes de distintos marcos teóricos. En particular, este trabajo toma argumentos provenientes de dos enfoques: i) nueva geografía económica (Krugman, 1991, 1995, 1998 y1999) y ii) recursos y capacidades (Penrose, 1959; Teece, 1980; Montgomery, 1994).

\section{Nueva geografía económica}

Este enfoque se compone de un conjunto de modelos de equilibrio general en una estructura de competencia imperfecta que consideran la existencia de rendimientos crecientes a escala, costos de transporte y la posibilidad de desplazamiento territorial de los factores productivos y de los consumidores (Krugman, 1991; Fujita y Krugman, 2004). Si bien estos modelos generan una gran variedad de resultados dependiendo de los supuestos específicos, la nueva geografía económica resalta una serie de elementos que pueden explicar la diversidad productiva en las grandes aglomeraciones (Bishop y Gripaios, 2007).

Krugman (1998) plantea la existencia de dos fuerzas contrapuestas: las centrípetas (que concentran geográficamente la actividad económica) y las centrífugas (que la disipan). Las primeras se derivan de una causalidad circular: por un lado, los trabajadores encuentran en los grandes centros urbanos infraestructuras de mejor calidad, salarios reales más elevados y una mayor variedad de bienes, mientras que por otro lado las empresas se localizan en grandes mercados para explotar economías de escala internas y externas. Asimismo, en el concepto de economías externas se suele distinguir entre las fuerzas de localización y las de urbanización. Las primeras se refieren a los beneficios derivados de la proximidad de empresas del mismo sector industrial (mercado de trabajo especializado, proveedores y efectos de derrame tecnológico, conocidos como spillovers), mientras que las segundas son independientes del sector industrial (servicios públicos, financieros, comerciales, transferencia de conocimiento) (Hoover, Jr., 1936). La evidencia indica que las economías de localización revisten especial importancia para las manufacturas tradicionales, mientras que las de urbanización ejercen mayor impacto en los servicios y en las manufacturas de alta tecnología (Henderson, Kuncoro y Turner, 1995). En este sentido, un entorno más urbanizado, proclive a la transmisión de conocimientos e innovación, será más propicio para la diversidad productiva (Glaeser y otros, 1992; McCann y Van Oort, 2009).

\section{Recursos y capacidades regionales}

El enfoque basado en los recursos (Resource-based view) surge a partir del aporte de Penrose (1959) al análisis de las empresas. Bajo esta óptica, las empresas se analizan sobre la base de sus recursos "heredados" (Teece, 1980; Montgomery, 1994). Esta teoría también se aplica a las regiones, que pueden ser definidas por su dotación de recursos tangibles (como la infraestructura) e intangibles (como el capital humano de sus trabajadores o la calidad de sus instituciones) (Lawson, 1999; Boschma, 2004; Neffke y otros, 2014; Boschma y Capone, 2015). Dado que la dotación de recursos 
y las capacidades condicionan las actividades productivas que pueden llevar a cabo las empresas de una región, es preciso incluirlos como un factor asociado al grado y a la evolución de la diversidad productiva regional.

En esta línea, un enfoque más moderno propuesto por Hidalgo y otros (2007) analiza la red de relaciones entre productos e industrias, originadas en vínculos de insumo-producto, sofisticación tecnológica y utilización de capacidades o infraestructuras similares. De acuerdo con este enfoque, el desarrollo de mayores capacidades, entendidas estas en un sentido amplio (productivas, organizativas e institucionales, entre otras) permite ampliar el abanico productivo e incluso producir bienes de mayor complejidad. Es por eso que los países tienden a diversificarse hacia productos cercanos a los que ya exportan, es decir, bienes que requieren capacidades similares. A nivel regional, el proceso de diversificación también tiende a ocurrir en actividades relacionadas con las ya existentes (Neffke, Henning y Boschma, 2011) y se ve afectado por la historia industrial local, las capacidades acumuladas en el territorio y el sendero previo (path-dependence) transitado por la región (Martin y Sunley, 2006; Neffke y otros, 2014).

\section{Antecedentes empíricos}

El tema de la diversidad productiva ha sido objeto de estudio de múltiples disciplinas y paradigmas (Ramanujan y Varadarajan, 1989). En este sentido, existen al menos tres dimensiones de análisis: empresa, región y país. En particular, los trabajos a nivel regional han experimentado un notable crecimiento en los últimos años (Frenken y otros, 2004), especialmente en los países desarrollados ${ }^{3}$.

La evidencia empírica indica que la diversidad productiva de una región está positivamente relacionada con su tamaño (Duranton y Puga, 2000; Monastiriotis, 2000; Beckstead y Brown, 2003; Bishop y Gripaios, 2007) y el grado de urbanización (Dewhurst y McCann, 2002; Bishop y Gripaios, 2007). Es decir, tal como es de esperar de acuerdo con los elementos señalados por la nueva geografía económica, los núcleos urbanos de mayor tamaño y con mayor densidad poblacional son también los más diversos.

Por otro lado, los trabajos empíricos relacionados con el enfoque de las capacidades y los recursos se enfrentan con la dificultad de identificarlos adecuadamente. Las contribuciones existentes cuantifican las capacidades regionales a partir de la incorporación de variables sustitutas (proxy) como la intensidad en investigación y desarrollo (I+D) (Aw y Batra, 1998; Parteka y Tamberi, 2011), la proporción de gerentes o responsables técnicos en el total del empleo industrial (Baldwin y otros, 2000), el número de empleados en industrias basadas en ciencia y técnica (Baldwin y otros, 2000; Bishop y Gripaios, 2007) o el nivel educativo de la población (Parteka y Tamberi, 2011).

Por otra parte, algunas contribuciones empíricas vinculan el grado de diversidad de una región o país con su nivel de desarrollo, medido en términos del producto bruto per cápita. En esta línea, De Benedictis, Gallegati y Tamberi (2009) y Parteka y Tamberi (2011) llegan a la conclusión de que las regiones con menor nivel de producto per cápita poseen un menor grado de heterogeneidad productiva relativa. Al respecto, Imbs y Wacziarg (2003) demuestran que la relación entre el producto per cápita y la diversidad a nivel de países es no lineal, es decir, hasta que los países alcanzan determinados niveles de ingreso el crecimiento se asocia positivamente a una estructura productiva y exportadora más diversa, pero luego la relación se revierte y comienzan a especializarse.

\footnotetext{
3 Por ejemplo, Beckstead y Brown (2003) en el Canadá; Rodgers (1957); Monastiriotis (2000) y Essletzbichler y Rigby (2007) en los Estados Unidos; Boschma, Minondo y Navarro (2013) en España; Bishop y Gripaios (2007) en el Reino Unido y Boschma e lammarino (2007) y Marra, Carlei y Paglialunga (2011) en Italia. El número de investigaciones empíricas en países en desarrollo es reducido, debido a la menor calidad y disponibilidad de los datos (Nachum, 2004; Hammouda y otros, 2006).
} 
Un último grupo de trabajos explica los cambios en el grado de diversidad productiva regional a partir de elementos relacionados con la dinámica empresarial. En este sentido, señalan que un aumento de la diversidad regional puede manifestarse a partir de diferentes fenómenos:

- la creación de nuevas empresas en ramas de actividad existentes pero con menor participación relativa en el empleo regional (ramas "no tradicionales"), o el crecimiento de las empresas existentes en esas ramas (Neffke, Henning y Boschma, 2011);

- la salida de empresas o la contracción del empleo en ramas con mayor participación relativa en el total de ocupados de la región (ramas "tradicionales");

- el ingreso de nuevas ramas de actividad productiva (Kamien y Schwartz, 1975).

En síntesis, a partir de lo expuesto se espera que la diversidad productiva regional se asocie positivamente con el grado de desarrollo regional, el tamaño de la región, una mayor urbanización y el nivel de capacidades existentes en el territorio. Asimismo, se espera que la diversidad productiva se asocie con algunas variables de dinámica empresarial (entrada y salida de empresas), aunque no es posible establecer a priori el sentido de dicha relación.

\section{Metodología}

\section{Fuente de datos}

La principal fuente de información es la Base de Datos para el Análisis Dinámico del Empleo (BADE), elaborada por el Observatorio de Empleo y Dinámica Empresarial (OEDE), que depende del Ministerio de Trabajo, Empleo y Seguridad Social de la Nación (MTEySS). La unidad de análisis es la empresa, que es identificada por su Código Único de Identificación Tributaria (CUIT). La base se construye a partir de las declaraciones de las empresas al Sistema Integrado de Jubilaciones y Pensiones, por lo cual contiene datos del total del empleo registrado asalariado en el sector privado en la Argentina (Castillo y otros, 2004). Se trabaja a partir de datos de empleo industrial desagregados a nivel sectorial a dos dígitos de la Clasificación Industrial Internacional Uniforme (CIIU), Rev. 3.1 (véase el anexo A1) y a nivel regional en las 24 jurisdicciones que componen el territorio argentino (23 provincias y la Ciudad Autónoma de Buenos Aires).

Para caracterizar a las provincias se consultaron además diversas fuentes de datos: el Censo Nacional de Población y Vivienda, anuarios estadísticos del Instituto Nacional de Estadísticas y Censos (INDEC), direcciones de estadísticas provinciales y datos publicados por el Ministerio de Industria y el Ministerio de Ciencia y Tecnología, así como los correspondientes a la propia BADE.

\section{2. Índice de diversidad productiva}

La utilización de índices de diversidad regional permite sintetizar gran cantidad de información e interpretarla fácilmente. Como es usual en la literatura especializada (Aw y Batra, 1998; Duranton y Puga, 2000; Hammouda y otros, 2006; Klinger y Lederman, 2004; Parteka y Tamberi (2011); Cadot, Carrère y Strauss-Kahn, 2011), en este trabajo se emplea como medida de diversidad la inversa del índice de Herfindahl-Hirschman $(\mathrm{HH})$, construida a partir de datos del empleo regional registrado asalariado en la industria manufacturera ${ }^{4}$. El índice $\mathrm{HH}$ se define como la sumatoria de la participación

\footnotetext{
${ }^{4}$ Alternativamente, el índice puede calcularse a partir de datos de producto o de valor agregado, pero no es posible contar con este tipo de información a nivel regional en la Argentina.
} 
de cada rama en el empleo regional, elevada al cuadrado (Duranton y Puga, 2000). Su inversa sería la siguiente:

$$
D P_{i}=1 / H H=1 / \sum_{j=1}^{J}\left(\frac{E_{i}^{j}}{E_{i}}\right)^{2}
$$

donde $E_{i}^{j}$ es el número de empleados en la rama $j$ en la región $i$ y $E_{i}$ es el número total de empleados industriales en la región.

\section{Modelo}

A fin de examinar la relación entre la diversidad productiva de la manufactura y los factores económicos asociados se estima un modelo econométrico de datos de panel. Una de las principales ventajas de su utilización consiste en el control de la heterogeneidad, tanto entre individuos como en el tiempo (Baltagi, 2008). Así, es posible controlar algunas características de las provincias (observables o no) que no cambian mucho a lo largo del período (por ejemplo, la dotación de recursos naturales o el entorno institucional).

La especificación del modelo se presenta en la ecuación (2), en la cual se incluyen efectos fijos por provincia y variables ficticias (dummies) por año para captar efectos temporales $\left(\theta_{t}\right)$. La variable dependiente $\left(D P_{i t}\right)$ es el índice de diversidad productiva definido en la ecuación (1).

$$
\begin{gathered}
D P_{i t}=\beta_{0}+\beta_{1} P P B G p c_{i t}+\beta_{2} \text { Densidad }_{i t}+\beta_{3} \text { Gasto en I\& }_{i t}+\beta_{4} P_{-} \text {expo }_{i t} \\
+\beta_{5} \text { Entrada }_{i t}-\beta_{6} \text { Salida }_{i t}+\beta_{t} \theta_{t}+\varepsilon_{i t}
\end{gathered}
$$

Los subíndices se refieren a la región $i$ y al tiempo $t$. En el cuadro 1 se describen las variables explicativas del modelo, su signo esperado y la fuente de datos. Dicha información se sintetiza en el cuadro 1, mientras que la matriz de correlación figura en el anexo A2.

\section{Cuadro 1}

\begin{tabular}{|c|c|c|c|c|c|c|c|c|}
\hline \multirow{2}{*}{ Factor asociado } & \multirow{2}{*}{ Variable } & & \multirow{2}{*}{$\begin{array}{c}\text { Signo } \\
\text { esperado } \\
\text { Media }\end{array}$} & \multirow{2}{*}{$\begin{array}{l}\text { Fuente } \\
\text { de datos } \\
\text { Desvío }\end{array}$} & \multicolumn{4}{|c|}{ Estadísticas descriptivas } \\
\hline & & & & & Media & Desvío & Mínimo & Máximo \\
\hline Desarrollo & $\begin{array}{l}\text { Producto bruto geográfico per } \\
\text { cápita (en millones de pesos a } \\
\text { valores constantes de 1993) }\end{array}$ & PBGpc & + & $\begin{array}{l}\text { CEP } \\
\text { EP }\end{array}$ & 0,007 & 0,005 & 0,002 & 0,034 \\
\hline $\begin{array}{l}\text { Grado de } \\
\text { urbanización }\end{array}$ & Densidad poblacional & Densidad & + & INDEC & 0,581 & 1,843 & 0,015 & 11,888 \\
\hline \multirow[t]{2}{*}{$\begin{array}{l}\text { Recursos y } \\
\text { capacidades }\end{array}$} & $\begin{array}{l}\text { Gasto público en investigación } \\
\text { y desarrollo (valores } \\
\text { corrientes - logaritmo) }\end{array}$ & Gasto_1+D & + & INDEC & 10,535 & 1,504 & 6,968 & 15,481 \\
\hline & Participación en las exportaciones & P_expo & + & INDEC & 3,978 & 8,010 & 0,036 & 39,447 \\
\hline \multirow{2}{*}{$\begin{array}{l}\text { Dinámica } \\
\text { empresarial }\end{array}$} & $\begin{array}{l}\text { Entrada de empresas industriales } \\
\text { (cantidad de empresas) }\end{array}$ & Entrada & $+/-$ & OEDE & 199,394 & 415,620 & 2,000 & 2946,00 \\
\hline & $\begin{array}{l}\text { Salida de empresas industriales } \\
\text { (cantidad de empresas) }\end{array}$ & Salida & $+/-$ & OEDE & 180,666 & 392,213 & 4,000 & 2540,00 \\
\hline
\end{tabular}

Argentina: factores asociados a la diversidad productiva regional: definición, fuente de datos, signo esperado y estadísticas descriptivas

Fuente: Elaboración propia.

Nota: CEP: Centro de Estudios para la Producción; EP: direcciones de estadísticas provinciales; INDEC: Instituto de Estadísticas y Censos; OEDE: Observatorio de Empleo y Dinámica Empresarial.

En primer lugar, se espera que el grado de desarrollo de una región, estimado a partir del producto bruto geográfico per cápita (PBGpc) se relacione positivamente con su diversidad productiva (De 
Benedictis, Gallegati y Tamberi, 2009; Parteka y Tamberi, 2011). Se trabaja con datos de PBG provincial publicados por el Centro de Estudios para la Producción (Ministerio de Industria de la Nación) y por las direcciones de estadísticas provinciales. Los datos son deflactados con el índice de precios implícitos (IPI) de la Argentina y con el índice de precios implícitos de la provincia de Buenos Aires (en el análisis de robustez), dado que no existen datos de PBGpc a precios constantes (ni índices de precios) para todas las provincias a lo largo del período analizado.

Además, a los efectos de evaluar la relación entre el grado de diversidad y la urbanización de cada región se incorpora la variable densidad, medida como el cociente entre la población de cada provincia y su superficie en $\mathrm{km}^{2}$. Como medida de las capacidades regionales se incluye, por un lado, el gasto público en investigación y desarrollo (gasto en I+D) y, por otro, el porcentaje de las exportaciones provinciales en el total del país (P_expo). Siguiendo a Castellacci (2007), Filipescu y otros (2013) y Artopoulos, Friel y Hallak (2013), se entiende que la inserción en el mercado externo puede ser consecuencia del desarrollo de capacidades productivas, organizacionales o institucionales. Así, una región orientada al mercado internacional requiere de un mayor aprendizaje y sofisticación, tanto de sus empresas exportadoras como de sus proveedores locales, para ajustarse a las características de la nueva demanda. El estímulo de la demanda externa también puede incentivar el desarrollo de nuevos productos y procesos, impulsando la diversidad a través de un "tirón de la demanda" (demand-pull) (Schmookler, 1966; Crépon, Duguet y Mairesse, 1998).

Por último, no es posible establecer a priori el sentido de la asociación entre el grado de diversidad y las variables de dinámica empresarial (cantidad de empresas industriales que nacen o mueren a lo largo del año). La diversidad se asociará en forma positiva a la entrada de empresas si estas ingresan en sectores menos tradicionales, y lo opuesto ocurrirá si el ingreso se produce en actividades tradicionales, es decir, en aquellas que concentran una alta participación del empleo regional (Dumais, Ellison y Glaeser, 2002; Noseleit, 2010). Por el contrario, la asociación entre la salida de empresas y la diversidad será negativa (positiva) si el cierre se produce en los sectores menos (más) tradicionales en cada región (Duranton y Puga, 2000; Noseleit, 2010). Los datos acerca de la dinámica empresarial provienen de la BADE, publicada por el OEDE.

\section{La industria manufacturera en la Argentina}

La industria manufacturera en la Argentina representa el 67\% de las exportaciones (33\% de origen agropecuario y $34 \%$ de origen industrial), el $22,5 \%$ del PIB y el $20 \%$ del empleo registrado en 2012 . A nivel regional pueden distinguirse diferentes perfiles industriales, de acuerdo a la importancia relativa de la industria en cada provincia y el tipo de especialización (véase el cuadro 2).

En las provincias que constituyen el principal núcleo productivo y de consumo del país (Buenos Aires, Santa Fe y Córdoba) la industria es, en términos de empleo, relativamente más importante: la participación del empleo industrial en estas jurisdicciones supera al promedio nacional (20\%). La Ciudad Autónoma de Buenos Aires (CABA), por su parte, se especializa en servicios debido principalmente a las deseconomías de aglomeración urbana existentes para la producción industrial. En estas jurisdicciones, al igual que para el resto de las provincias del país (salvo Chaco y Tierra del Fuego), la principal rama es la elaboración de alimentos y bebidas (división 15). Le siguen en orden de importancia industrias vinculadas a la población (como edición e imprenta -división 22-) o al sector agropecuario (maquinarias y equipos -división 29-). 
Cuadro 2

Argentina: participación del sector industrial en el total del empleo provincial

\begin{tabular}{|c|c|c|c|c|c|c|}
\hline \multirow[t]{2}{*}{ Grupos provinciales } & \multirow[t]{2}{*}{ Provincia } & \multicolumn{2}{|c|}{$\begin{array}{l}\text { Porcentaje de } \\
\text { empleo industrial }\end{array}$} & \multicolumn{3}{|c|}{ 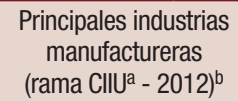 } \\
\hline & & 1996 & 2012 & $1^{0}$ & $2^{0}$ & $3^{\circ}$ \\
\hline \multirow[t]{4}{*}{ 1. Núcleo productivo y de consumo } & Ciudad Autónoma de Buenos Aires & 18,8 & 13,7 & 15 & 26 & 22 \\
\hline & Buenos Aires & 33,6 & 26,5 & 15 & 24 & 25 \\
\hline & Santa Fe & 31,0 & 26,1 & 15 & 29 & 28 \\
\hline & Córdoba & 26,7 & 21,1 & 15 & 34 & 29 \\
\hline \multirow[t]{5}{*}{ 2. Con promoción industrial } & San Luis & 51,9 & 34,7 & 15 & 17 & 25 \\
\hline & San Juan & 26,6 & 17,3 & 15 & 26 & 24 \\
\hline & Catamarca & 27,1 & 21,5 & 15 & 17 & 18 \\
\hline & La Rioja & 44,1 & 33,9 & 15 & 17 & 19 \\
\hline & Tierra del Fuego & 28,4 & 36,9 & 32 & 34 & 25 \\
\hline \multirow[t]{8}{*}{ 3. Complejo agroindustrial } & Tucumán & 25,6 & 16,0 & 15 & 17 & 28 \\
\hline & La Pampa & 13,9 & 11,5 & 15 & 17 & 26 \\
\hline & Entre Ríos & 21,1 & 18,8 & 15 & 20 & 28 \\
\hline & Salta & 16,0 & 14,2 & 15 & 16 & 24 \\
\hline & Río Negro & 10,6 & 8,9 & 15 & 24 & 26 \\
\hline & Mendoza & 25,3 & 19,3 & 15 & 26 & 29 \\
\hline & Jujuy & 29,8 & 22,0 & 15 & 27 & 16 \\
\hline & Santiago del Estero & 15,4 & 10,7 & 15 & 17 & 26 \\
\hline \multirow[t]{3}{*}{ 4. Complejos petrolero y pesquero } & Neuquén & 11,3 & 7,9 & 15 & 28 & 29 \\
\hline & Chubut & 17,3 & 11,6 & 15 & 17 & 27 \\
\hline & Santa Cruz & 6,5 & 5,4 & 15 & 29 & 28 \\
\hline \multirow[t]{4}{*}{ 5. Complejos forestal y textil } & Chaco & 16,9 & 12,0 & 15 & 17 & 26 \\
\hline & Formosa & 11,6 & 7,3 & 15 & 24 & 36 \\
\hline & Corrientes & 18,4 & 15,1 & 15 & 20 & 17 \\
\hline & Misiones & 30,5 & 21,2 & 15 & 20 & 21 \\
\hline
\end{tabular}

Fuente: Elaboración propia, sobre la base de la Base de Datos para el Análisis Dinámico del Empleo (BADE).

a Clasificación Industrial Internacional Uniforme (CIIU), Rev. 3.1.

b En términos de empleo.

Otro conjunto de provincias con actividad industrial superior a la media nacional está conformado por las regiones beneficiadas con regímenes de promoción industrial (Tierra del Fuego, San Luis, La Rioja y Catamarca) $)^{5}$. En estas provincias, las actividades con mayor peso relativo son aquellas favorecidas por las políticas de promoción (como la fabricación de equipos y aparatos de radio, televisión y comunicaciones -división 32- en Tierra del Fuego o la fabricación de productos textiles -división 17-).

Un tercer grupo de provincias presenta un perfil netamente agroindustrial (Tucumán, La Pampa, Entre Ríos, Salta, Río Negro, Mendoza, Jujuy y Santiago del Estero). En ellas, la elaboración de alimentos y bebidas concentra más del $50 \%$ del empleo industrial registrado. Por su parte, Neuquén, Chubut y Santa Cruz se caracterizan por la realización de actividades relacionadas con los complejos petrolero y pesquero. Por último, un quinto grupo está compuesto por provincias cuya industria (aserraderos -grupo 20-, muebles -división 36-y textiles -división 17-) se vincula a los recursos naturales existentes en la región (Chaco, Corrientes, Formosa y Misiones).

5 Las provincias que cuentan con regímenes de promoción industrial son La Rioja (Ley 22.021/79), San Luis y Catamarca (Ley 22.702/82) y San Juan (Ley 22.973). Por su parte, Tierra del Fuego es considerada como un Área Aduanera Especial (Ley 19.640/72). 


\section{Resultados}

\section{Diversidad productiva de la industria a nivel nacional y regional (1996-2012)}

La diversidad productiva de la industria argentina se relaciona estrechamente con las fluctuaciones macroeconómicas de los últimos 20 años, que afectaron a la economía en general y a la industria manufacturera en particular. En el gráfico 1 se observa que la diversidad industrial es un fenómeno claramente procíclico, es decir, evoluciona de forma similar al producto (medido en el eje derecho). El indicador de diversidad desciende en forma continua desde 1998 hasta llegar a un mínimo en 2002, en coincidencia con la crisis política, económica y social desencadenada en la Argentina a finales de 2001. En la etapa de recuperación económica iniciada en 2003 la diversidad industrial aumenta en forma continua, incluso por encima de los valores previos a la crisis, alcanzando su valor máximo en 2008. A partir de ese año disminuye nuevamente, en concordancia con la crisis financiera internacional y la apreciación gradual del tipo de cambio, sin llegar a recuperarse en períodos posteriores. La asociación positiva entre el indicador de diversidad industrial y el producto interno bruto a precios de mercado (PIBpm) es estadísticamente significativa, obteniéndose resultados análogos para la tasa de crecimiento del PIBpm (véase el cuadro 3).

\section{Gráfico 1}

Argentina: evolución de la diversidad industrial (1/HH) y del producto interno bruto a precios de mercado (PBIpm), 1996-2012

(En millones de pesos a precios constantes de 1993)

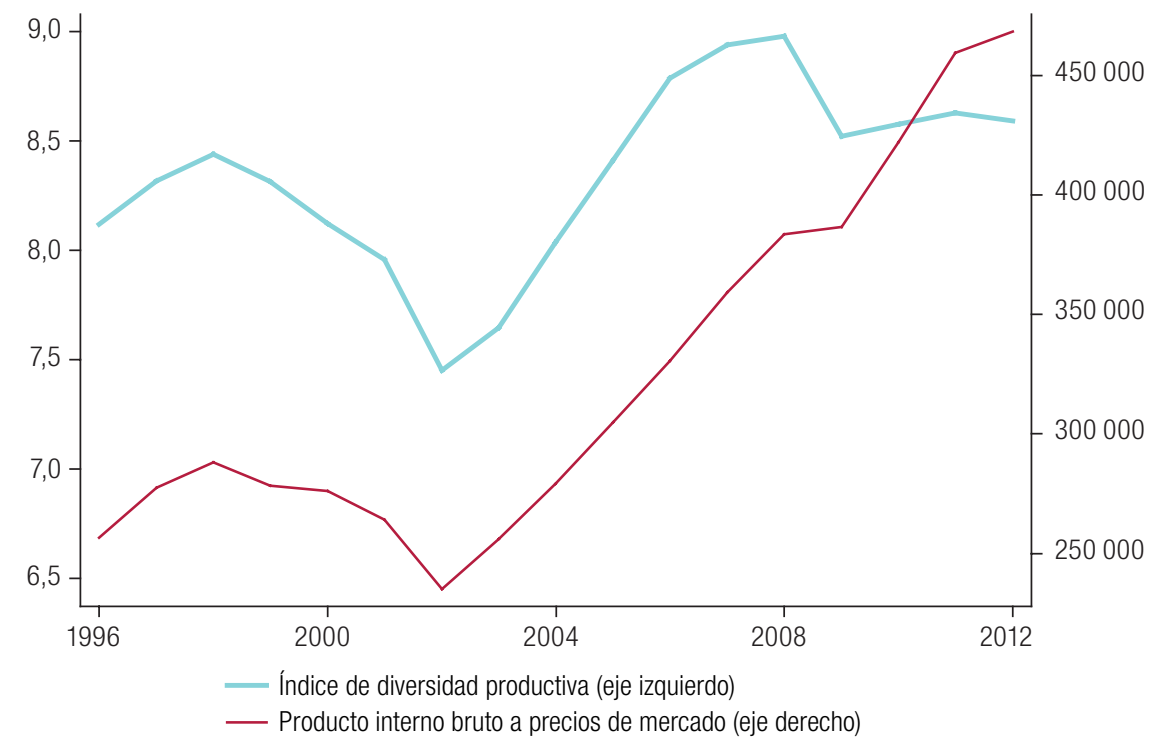

Fuente: Elaboración propia, sobre la base de Instituto Nacional de Estadísticas y Censos (INDEC) y de la Base de Datos para el Análisis Dinámico del Empleo (BADE).

Nota: HH: Herfindahl-Hirschman.

\footnotetext{
6 A partir de 2007 se interrumpe la asociación marcadamente positiva entre ambas variables. Es posible que esto obedezca a una sobreestimación del PIB en términos reales como consecuencia de la intervención en ese año del organismo oficial de estadísticas (INDEC).
} 


\section{Cuadro 3}

Argentina: correlación entre el producto interno bruto a precios de mercado (PIBpm) y el índice de diversidad

\begin{tabular}{lc}
\hline Variables & $\begin{array}{c}\text { Coeficiente de correlación } \\
\text { de Pearson }\end{array}$ \\
\hline PIBpm/Índice de diversidad & $0,746^{*}$ \\
\hline Tasa de crecimiento del PIBpm/Índice de diversidad & $0,551^{* *}$ \\
\hline Fuente: Elaboración propia, sobre la base de Instituto Nacional de Estadísticas y Censos \\
$\begin{array}{l}\text { (INDEC) y de la Base de Datos para el Análisis Dinámico del Empleo (BADE). } \\
\text { Nota: }\end{array} \quad$ Relaciones estadísticamente significativas: ${ }^{* \star} 0,05 ;{ }^{*} 0,10$.
\end{tabular}

Ahora bien, en el interior de la Argentina, ¿la diversidad también es un fenómeno procíclico en todas las regiones? ¿Todas las provincias son igualmente diversas? ¿Qué factores económicos se pueden asociar a un mayor o menor grado de diversidad productiva industrial?

Lejos de ser homogéneo, el territorio argentino presenta una importante heterogeneidad estructural, que se manifiesta en una tradicional desigualdad económica y territorial, que a su vez obedece a la diversidad de recursos, la densidad poblacional, la localización industrial y la urbanización de determinadas áreas geográficas (Zalduendo, 1975; Gatto, 2007). Estas desigualdades también se observan en materia de diversidad productiva industrial (véase el mapa 1).

Mapa 1

Argentina: grado de diversidad productiva (1/HH), 2012

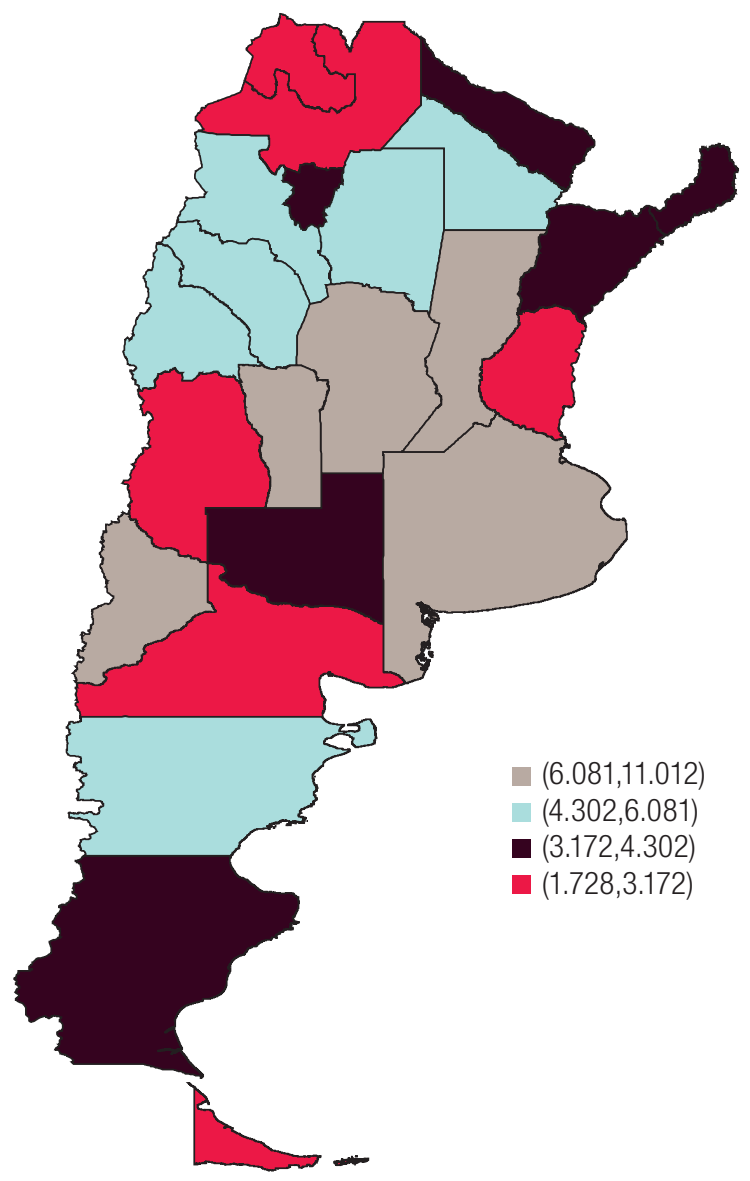

Fuente: Elaboración propia, sobre la base de la Base de Datos para el Análisis Dinámico del Empleo (BADE). 
Por un lado, se observa que las principales jurisdicciones del país (Ciudad Autónoma de Buenos Aires, y las provincias de Buenos Aires, Santa Fe y Córdoba) son las más diversas, junto a San Luis y Neuquén. En contraste, algunas provincias agroindustriales (Salta, Jujuy, Entre Ríos, Mendoza y Río Negro) son las menos diversas, ya que la industria alimenticia ocupa a alrededor del $60 \%$ del empleo industrial ${ }^{7}$. Algo similar sucede en Tierra del Fuego, donde la industria de fabricación de aparatos de radio y televisión representa el 60\% del empleo industrial.

La elevada diversidad de la Ciudad Autónoma de Buenos Aires y de las provincias centrales (Buenos Aires, Santa Fe y Córdoba) ha sido un rasgo estructural y relativamente estable en los últimos años (véase el cuadro 4). Por otra parte, en las provincias con regímenes de promoción industrial (San Luis, San Juan, La Rioja y Catamarca) la diversidad ha disminuido y esto podría deberse al impacto decreciente de dichos regímenes sobre los sectores menos tradicionales de cada región (Donato, 2007). En Tierra del Fuego también se observa una abrupta disminución de la diversidad a partir de 2009, a raíz del aumento relativo del empleo en los sectores beneficiados por regímenes especiales en esa etapa (radio y televisión, textil, cuero y calzado).

Cuadro 4

Argentina: evolución del índice de diversidad productiva provincial (1996-2012)

\begin{tabular}{|c|c|c|c|c|c|}
\hline \multirow{2}{*}{ Clasificación provincial } & \multirow{2}{*}{ Provincia } & \multirow{2}{*}{$\begin{array}{c}\text { Índice de } \\
\text { diversidad } \\
\text { productiva-1996 }\end{array}$} & \multirow{2}{*}{$\begin{array}{c}\text { Índice de } \\
\text { diversidad } \\
\text { productiva-2012 }\end{array}$} & \multirow{2}{*}{$\begin{array}{l}\text { Cambio entre } \\
1996 \text { y } 2012\end{array}$} & \multirow{2}{*}{$\begin{array}{c}\begin{array}{c}\text { Coeficiente de } \\
\text { variabilidad }\end{array} \\
(1996-2012)\end{array}$} \\
\hline & & & & & \\
\hline \multirow[t]{4}{*}{$\begin{array}{l}\text { Núcleo productivo } \\
\text { y de consumo }\end{array}$} & $\begin{array}{l}\text { Ciudad Autónoma de } \\
\text { Buenos Aires }\end{array}$ & 9,65 & 11,01 & $\uparrow$ & 0,07 \\
\hline & Buenos Aires & 10,29 & 10,14 & $\downarrow$ & 0,03 \\
\hline & Santa Fe & 5,73 & 6,99 & $\uparrow$ & 0,06 \\
\hline & Córdoba & 5,57 & 6,09 & $\uparrow$ & 0,06 \\
\hline \multirow{5}{*}{$\begin{array}{l}\text { Con promoción } \\
\text { industrial }\end{array}$} & San Luis & 10,45 & 7,52 & $\downarrow$ & 0,09 \\
\hline & San Juan & 5,55 & 5,94 & $\uparrow$ & 0,04 \\
\hline & Catamarca & 6,58 & 5,41 & $\downarrow$ & 0,08 \\
\hline & La Rioja & 5,68 & 5,19 & $\downarrow$ & 0,05 \\
\hline & Tierra del Fuego & 4,33 & 2,57 & $\downarrow$ & 0,15 \\
\hline \multirow{7}{*}{$\begin{array}{l}\text { Complejo } \\
\text { agro-industrial }\end{array}$} & Tucumán & 3,43 & 3,44 & $\uparrow$ & 0,03 \\
\hline & La Pampa & 4,68 & 3,23 & $\downarrow$ & 0,17 \\
\hline & Entre Ríos & 2,61 & 3,10 & $\uparrow$ & 0,08 \\
\hline & Salta & 2,75 & 2,77 & $\uparrow$ & 0,04 \\
\hline & Río Negro & 2,53 & 2,54 & $\uparrow$ & 0,09 \\
\hline & Mendoza & 2,45 & 2,33 & $\downarrow$ & 0,06 \\
\hline & Jujuy & 2,27 & 1,72 & $\downarrow$ & 0,11 \\
\hline \multirow{3}{*}{$\begin{array}{l}\text { Complejos } \\
\text { petrolero y pesquero }\end{array}$} & Neuquén & 6,57 & 6,58 & $\uparrow$ & 0,04 \\
\hline & Chubut & 4,59 & 5,98 & $\uparrow$ & 0,10 \\
\hline & Santa Cruz & 5,25 & 4,06 & $\downarrow$ & 0,15 \\
\hline \multirow{5}{*}{$\begin{array}{l}\text { Complejos } \\
\text { forestal y textil }\end{array}$} & Santiago del Estero & 4,28 & 4,43 & $\uparrow$ & 0,04 \\
\hline & Chaco & 4,55 & 6,06 & $\uparrow$ & 0,08 \\
\hline & Formosa & 4,85 & 4,17 & $\downarrow$ & 0,13 \\
\hline & Corrientes & 3,74 & 4,06 & $\uparrow$ & 0,08 \\
\hline & Misiones & 3,88 & 4,00 & $\uparrow$ & 0,05 \\
\hline
\end{tabular}

Fuente: Elaboración propia, sobre la base de la Base de Datos para el Análisis Dinámico del Empleo (BADE).

\footnotetext{
7 Teniendo en cuenta el grado de desarrollo productivo de algunas provincias (como Mendoza o Entre Ríos), es posible que exista una alta diversidad al interior de la rama alimentos (diversidad relacionada), que no puede ser captada por el indicador de diversidad utilizado (inversa del índice Herfindahl-Hirschman $(\mathrm{HH})$ con datos de empleo desagregados a dos dígitos de la Clasificación Industrial Internacional Uniforme (CIIU).
} 
En el gráfico 2 se muestra la evolución de la diversidad promedio en función de la agrupación provincial presentada en el cuadro 4, junto con el índice a nivel nacional. Se observa que la tendencia nacional la establecen las regiones más diversas que, justamente, son las de mayor peso relativo en términos de empleo. Los demás grupos muestran una tendencia similar, es decir, presentan un comportamiento procíclico, salvo las provincias favorecidas por políticas de promoción industrial, cuya diversidad disminuye en forma constante ${ }^{8}$.

\section{Gráfico 2}

Argentina: evolución de la diversidad industrial (1/HH) por grupos de provincias

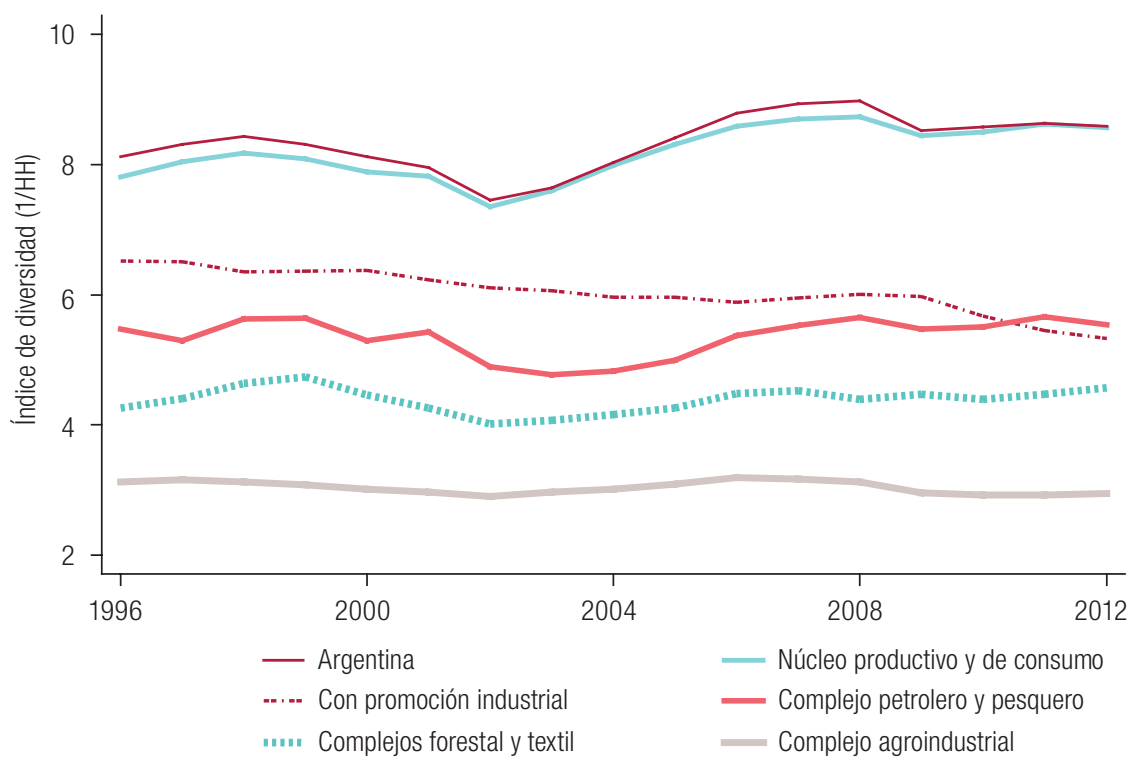

Fuente: Elaboración propia, sobre la base de la Base de Datos para el Análisis Dinámico del Empleo (BADE).

Cabe destacar que un aumento en el grado de diversidad puede asociarse alternativamente con dos fenómenos: o bien un crecimiento en el empleo de las ramas menos tradicionales (manteniendo o creciendo menos el empleo en las ramas tradicionales), o bien una disminución del empleo en las ramas principales. Un ejemplo del primer caso es la provincia de Chubut, donde el aumento en la diversidad se relaciona con el crecimiento de ramas poco tradicionales como productos de metal -división 28- o de minerales no metálicos -división 26-, mientras que Chaco es un ejemplo del segundo caso (el aumento de la diversidad se debe a la caída del empleo textil -división 17- (véase el gráfico 3). Teniendo en cuenta que tanto las causas como los efectos asociados a la diversidad posiblemente varíen en cada uno de los casos planteados, en futuras investigaciones sobre el tema debería tomarse este punto en consideración ${ }^{9}$.

\footnotetext{
8 En el anexo A3 se presenta la evolución del índice de diversidad productiva por provincia.

9 En los modelos de la sección VI.2 no fue posible incorporar este aspecto, ya que Chaco es la única provincia con un comportamiento asociado al segundo grupo. Por este motivo, no es posible estimar diferentes regresiones para cada grupo ni incorporar una variable binaria a las regresiones de datos de panel de efectos fijos.
} 


\section{Gráfico 3}

Argentina (Chubut y Chaco): participación de las ramas en el total de empleo industrial, 1996 y 2012

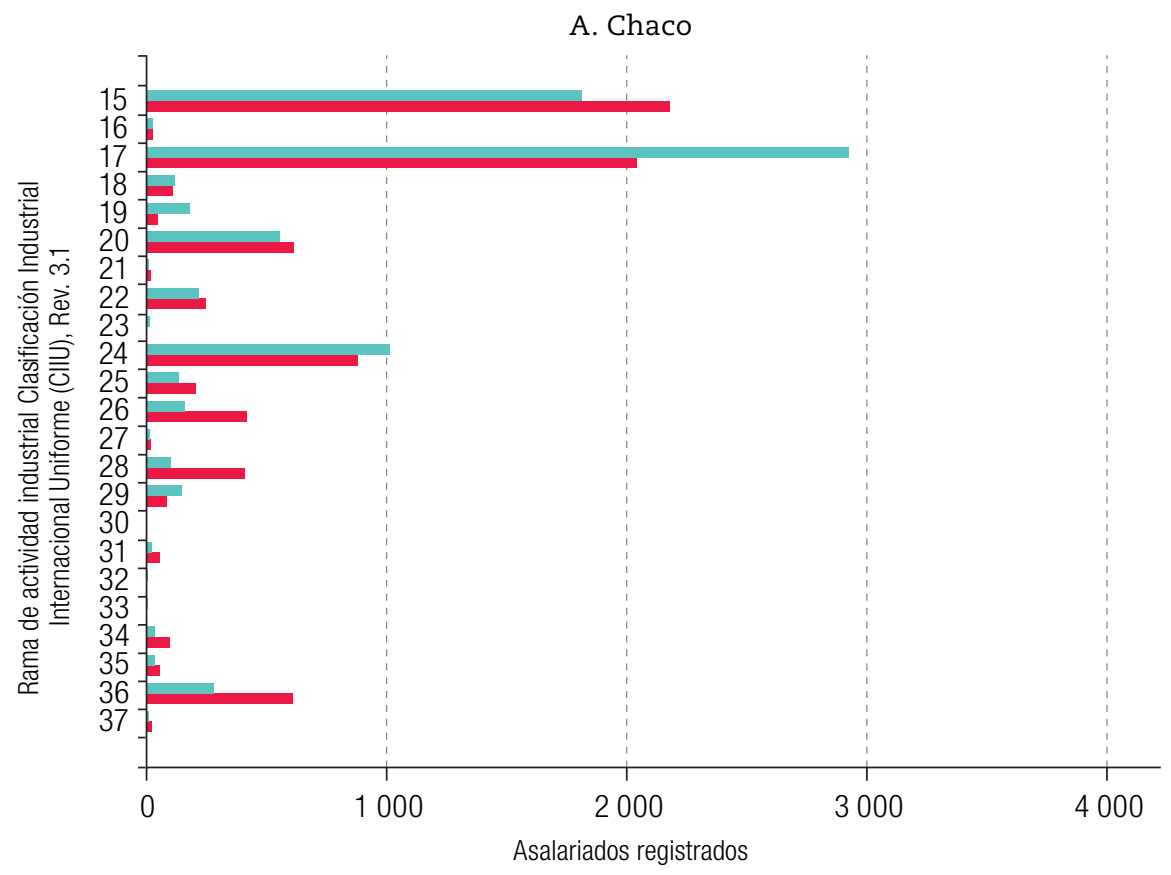

\section{B. Chubut}

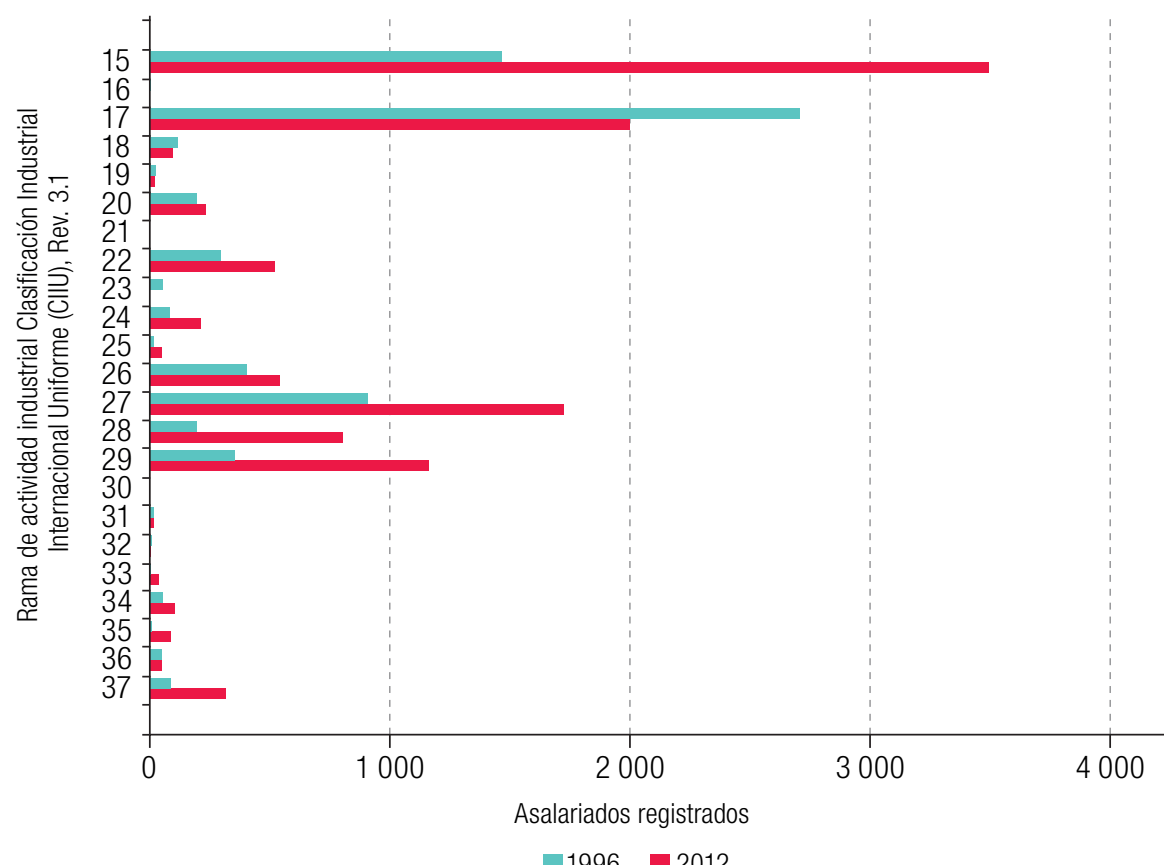

Fuente: Elaboración propia, sobre la base de la Base de Datos para el Análisis Dinámico del Empleo (BADE).

Nota: Solo se incluyen dos casos que contraponen las dos causas detalladas de aumento de la diversidad regional: i) el crecimiento del empleo en sectores menos tradicionales y ii) la disminución del empleo en sectores tradicionales. 


\section{Factores económicos asociados a la diversidad productiva regional}

Para identificar los factores económicos que se asocian al grado de diversidad regional se estima un modelo de datos de panel de efectos fijos (EF). Este modelo fue preferido al de efectos aleatorios ya que se rechaza la hipótesis nula del test de Hausman $(1978)^{10}$. En el cuadro 5 se presentan los resultados de la estimación, que incluyen variables ficticias (dummies) por año para captar los efectos temporales. Por su parte, las variables explicativas se toman rezagadas un período como estrategia ante el potencial problema de endogeneidad (Bebczuk y Berrettoni, 2006).

La mayor parte de los coeficientes estimados resultan estadísticamente significativos y con el signo esperado, de acuerdo con la literatura especializada. En primer lugar, existe una relación directa entre el producto per cápita de la región y la diversidad productiva. Es decir, se verifica que el grado de desarrollo regional se asocia positivamente con la diversidad productiva regional ${ }^{11}$.

\section{Cuadro 5}

Argentina: factores asociados a la diversidad de la industria regional (Modelo 1)

\begin{tabular}{lc}
\hline Factores & Modelo 1 \\
\hline Producto bruto geográfico (PBG) per cápita & $84,8968^{\star}$ \\
\hline Densidad & $(34,8276)$ \\
\hline Gasto en investigación y desarrollo (I+D) & $0,9827^{\star * *}$ \\
\hline Participación en las exportaciones & $(-0,1433)$ \\
\hline Ingreso de empresas industriales & 0,2095 \\
\hline Salida de empresas industriales & $(-0,1533)$ \\
\hline Constante & $0,0552^{*}$ \\
\hline Test F & $(-0,0211)$ \\
\hline R $^{2}$ (W) & 0,0000 \\
\hline
\end{tabular}

Fuente: Elaboración propia.

Notas: Significación: * $p<0,05 ;{ }^{* *} p<0,01 ;{ }^{* \star} \quad p<0,001 ; 378$ observaciones; efectos fijos por provincia, variables ficticias (dummies) por año; variable dependiente: índice de diversidad productiva; variables rezagadas un período; errores estándares robustos entre paréntesis.

La densidad poblacional (variable proxy del grado de urbanismo) se asocia de forma directa a la diversidad. Es decir, existe evidencia a favor de las economías de urbanización como factor asociado a la diversidad industrial regional. Se encuentra también una relación positiva y significativa entre la

\footnotetext{
${ }^{10}$ El supuesto clave implica que en el modelo de efectos aleatorios no puede asumirse que las variables explicativas sean independientes del término de error, por lo cual si se rechaza la hipótesis nula, se sugiere trabajar con un estimador de efectos fijos, que siempre proporciona estimaciones coherentes (Wooldridge, 2010). Las estimaciones con efectos aleatorios se encuentran disponibles para su consulta.

${ }^{11}$ En especificaciones alternativas se incluyó además el término cuadrático ( $\mathrm{PBGpc}$ ) para determinar si existe una relación no lineal entre diversidad regional y desarrollo, como la observada a nivel de países por Imbs y Wacziarg (2003). El término no resultó estadísticamente significativo. Esto podría deberse a que las regiones argentinas son mucho más homogéneas en términos de desarrollo que los países analizados por estos autores.
} 
participación de cada provincia en las exportaciones nacionales y su grado de diversidad, entendiéndose que las provincias con mayor inserción en el mercado externo poseen más capacidades y recursos, lo que aumenta las posibilidades de ampliar el abanico productivo de la industria local.

Por otro lado, si bien se esperaba que el gasto en investigación y desarrollo se asociara positivamente con la diversidad, el coeficiente resulta positivo pero no significativo. Esto puede obedecer a que el gasto público en investigación y desarrollo a nivel provincial puede no ser suficiente para fomentar un cambio en la estructura productiva en el caso de la Argentina.

Respecto de las variables que captan la dinámica industrial regional, se observa que la salida de empresas disminuye la diversidad. Esto implica que las salidas se producen mayormente en las ramas menos tradicionales de cada región, ya sea por contar con menos economías de localización, escasas capacidades productivas o institucionales para mantener a esas empresas en el mercado, o una infraestructura poco adecuada para su desarrollo. Por otro lado, la entrada de empresas no resulta estadísticamente significativa, lo cual puede deberse al ingreso de empresas tanto en ramas tradicionales como no tradicionales en cada región (compensándose el efecto de dichas entradas sobre la diversidad). Una mayor apertura de los datos de dinámica empresarial podría permitir identificar efectivamente en qué ramas de actividad se producen las entradas y salidas de empresas a nivel provincial y corroborar estas interpretaciones.

En el cuadro 6 se muestran los resultados de una especificación alternativa, que incluye una medida del tamaño de la región, a saber el producto bruto geográfico (PBG), a fin de analizar si, tal como sugiere la literatura especializada, las regiones de mayor tamaño son las más diversas (se omite la medida del grado de desarrollo (PBGpc) por alta multicolinealidad). Se observa que la relación entre el tamaño de la región y la diversidad es positiva y significativa, mientras que el resto de los resultados se mantienen.

\section{Cuadro 6}

Argentina: factores asociados a la diversidad de la industria regional (Modelo 2)

\begin{tabular}{lc}
\hline Factores & Modelo 2 \\
\hline Producto bruto geográfico (PBG) & $0,00001^{*}$ \\
\hline Densidad & $(0,000)$ \\
\hline Gasto en investigación y desarrollo (I+D) & $0,8387^{\star \star *}$ \\
\hline Participación en las exportaciones & $(0,118)$ \\
& 0,2237 \\
\hline Ingreso de empresas industriales & $(0,181)$ \\
\hline Salida de empresas industriales & $0,0600^{*}$ \\
\hline Constante & $(0,030)$ \\
\hline Test F & $-0,0001$ \\
\hline R 2 (W) & $(0,000)$ \\
\hline
\end{tabular}

Fuente: Elaboración propia.

Notas: Significación: * $p<0,05$; ** $p<0,01$; ${ }^{* \star *} p<0,001 ; 378$ observaciones; efectos fijos por provincia, variables ficticias (dummies) por año; variable dependiente: índice de diversidad productiva; variables rezagadas un período; errores estándares robustos entre paréntesis. 
Con el propósito de contrastar la robustez de los resultados, en el cuadro 7 se presentan diversas especificaciones alternativas de los modelos presentados. En primer lugar, dado que no existen índices de precios para todas las provincias, como alternativa al índice de precios implícitos (IPI) de la Argentina se toma el IPI de la Ciudad Autónoma de Buenos Aires, a fin de deflactar los datos de PBG (véase el cuadro 7). Se observa que los resultados se mantienen, utilizando al PBG tanto como medida de desarrollo (PBG per cápita-Modelo 3) como de proxy de tamaño (PBG-Modelo 4).

Cuadro 7

Argentina: factores asociados a la diversidad de la industria regional (Modelos 3 y 4)

\begin{tabular}{lcc}
\hline Factores & Modelo 3 & Modelo 4 \\
\hline Producto bruto geográfico (PBG) (deflactado con base en el índice de precios & $87,6756^{*}$ & \\
implícitos (IPI) de la Ciudad Autónoma de Buenos Aires) per cápita & $(32,6031)$ & $0,0001^{*}$ \\
\hline $\begin{array}{l}\text { PBG (deflactado con base en el índice de precios implícitos (IPI) } \\
\text { de la Ciudad Autónoma de Buenos Aires) }\end{array}$ & & $(0,000)$ \\
\hline Densidad & & $0,839^{* * *}$ \\
\cline { 2 - 3 } & & $(0,118)$ \\
\hline Gasto en investigación y desarrollo (I+D) & $(-0,1425)$ & 0,224 \\
\hline Participación en las exportaciones & 0,2134 & $(0,181)$ \\
\hline Ingreso de empresas industriales & $(-0,151)$ & $0,061^{*}$ \\
\hline Salida de empresas industriales & $0,0549^{*}$ & $(0,030)$ \\
\hline Constante & $(-0,0213)$ & 0,000 \\
\hline Test F & 0,0000 & $(0,000)$ \\
\hline $\mathrm{R}^{2}$ (w) & $(-0,0002)$ & $-0,001^{* *}$ \\
\hline
\end{tabular}

Fuente: Elaboración propia.

Notas: Significación: ${ }^{*} p<0,05 ;{ }^{\star \star} p<0,01 ;{ }^{\star \star \star} p<0,001 ; 378$ observaciones; efectos fijos por provincia, variables ficticias (dummies) por año; variable dependiente: índice de diversidad productiva; variables rezagadas un período; errores estándares robustos entre paréntesis.

Otra prueba de robustez consiste en estimar regresiones excluyendo las provincias cuya actividad principal concentra más del 50\% del empleo industrial regional (Tucumán, La Pampa, Entre Ríos, Salta, Río Negro, Mendoza, Jujuy y Tierra del Fuego). Es posible que en dichas provincias la evolución del índice se relacione con factores que afectan la evolución de la rama principal y no con aquellos que se vinculan con la diversidad. Al igual que en la regresión base estimada, la mayoría de las variables explicativas siguen siendo estadísticamente significativas y presentan el signo esperado (véase el cuadro 8). Se presentan los resultados tomando el producto bruto geográfico per cápita (PBGpc) constante (deflactado por el IPI de la Argentina (Modelo 5) y por el de la Ciudad Autónoma de Buenos Aires (Modelo 6)).

Por otro lado, a fin de controlar la posible existencia de un sendero previo (path dependence) en el proceso de diversificación, se incluye la variable dependiente rezagada un período como variable independiente y se modela a partir del estimador Arellano-Bond para paneles dinámicos. No obstante, a pesar de que el método generalizado de momentos (MGM) es globalmente significativo, las variables incorporadas no resultan estadísticamente significativas, lo cual puede ser prueba de multicolinealidad entre las variables mencionadas (véase el cuadro 9). Si bien la estimación por variables instrumentales podría ser adecuada en este caso, no es posible implementarla debido al escaso número de observaciones ${ }^{12}$.

12 Por ejemplo, He (2009) utiliza datos de 20.035 empresas; Elhiraika y Mbate (2014) trabajan con información de 53 países; Cadot, Carrère y Strauss-Kahn (2011) utilizan datos de exportaciones correspondientes a 79 países. 


\section{Cuadro 8}

Argentina: factores asociados a la diversidad de la industria regional (excluyendo provincias con empleo altamente concentrado)

\begin{tabular}{|c|c|c|}
\hline Factores & Modelo 5 & Modelo 6 \\
\hline \multirow{2}{*}{$\begin{array}{l}\text { Producto bruto geográfico (PBG) (deflactado con base en el índice } \\
\text { de precios implícitos (IPI) de la Argentina) per cápita }\end{array}$} & $101,409^{\star *}$ & \\
\hline & $(29,3258)$ & \\
\hline \multirow{2}{*}{$\begin{array}{l}\text { Producto bruto geográfico (PBG) (deflactado con base en el índice } \\
\text { de precios implícitos (IPI) de la Ciudad Autónoma de } \\
\text { Buenos Aires) per cápita }\end{array}$} & & $98,9882^{* \star}$ \\
\hline & & $(30,6885)$ \\
\hline \multirow[t]{2}{*}{ Densidad } & $4,6024^{\star \star}$ & $4,5532^{\star *}$ \\
\hline & $(1,4499)$ & $(1,5046)$ \\
\hline \multirow[t]{2}{*}{ Gasto en investigación y desarrollo (I+D) } & $0,4071^{*}$ & $0,4008^{*}$ \\
\hline & $(0,1698)$ & $(0,1712)$ \\
\hline \multirow[t]{2}{*}{ Participación en las exportaciones } & $0,0399^{*}$ & $0,0407^{*}$ \\
\hline & $(0,0208)$ & $(0,0206)$ \\
\hline \multirow[t]{2}{*}{ Ingreso de empresas industriales } & 0,0001 & 0,0001 \\
\hline & $(0,0002)$ & $(0,0002)$ \\
\hline \multirow[t]{2}{*}{ Salida de empresas industriales } & $-0,0007^{*}$ & $-0,0007^{*}$ \\
\hline & $(0,0003)$ & $(0,0003)$ \\
\hline \multirow[t]{2}{*}{ Constante } & 0,1987 & 0,2928 \\
\hline & $(1,9722)$ & $(1,9928)$ \\
\hline Test F & $6,96^{\star \star \star}$ & $9,28^{\star * *}$ \\
\hline$R^{2}(w)$ & 0,42 & 0,42 \\
\hline
\end{tabular}

Fuente: Elaboración propia.

Notas: Significación: * $p<0,05 ;{ }^{*} p<0,01 ;{ }^{* \star *} p<0,001 ; 256$ observaciones; efectos fijos por provincia, variables ficticias (dummies) por año; variable dependiente: índice de diversidad productiva; variables rezagadas un período; errores estándares robustos entre paréntesis.

\section{Cuadro 9}

Argentina: factores asociados a la diversidad de la industria regional (Modelo dinámico)

\begin{tabular}{lc}
\hline Factores & Modelo 7 \\
\hline Índice de diversidad (rezagado un período) & $-0,5481$ \\
\hline Producto bruto geográfico (PBG) per cápita & $(0,7465)$ \\
\hline Densidad & $-17,06$ \\
\hline \multirow{2}{*}{ Gasto en investigación y desarrollo (l+D) } & $(441,1592)$ \\
\hline Participación en las exportaciones & $-13,8913$ \\
\hline Ingreso de empresas industriales & $(14,1142)$ \\
\hline Salida de empresas industriales & 0,4415 \\
\hline Constante & $(0,9466)$ \\
\hline Test de Wald & $-0,0098$ \\
\hline
\end{tabular}

Fuente: Elaboración propia.

Notas: Significación: * $p<0,05$; ${ }^{\star \star} p<0,01$; ${ }^{* \star} p<0,001$ GMM; 378 observaciones; Rezagos (1) variable ficticia (dummy) por año; variable dependiente: índice de diversidad productiva; variables rezagadas un período; errores estándares robustos entre paréntesis. 


\section{VII.Reflexiones finales}

La literatura especializada considera que la diversidad productiva es un instrumento clave en el diseño de políticas industriales de desarrollo regional. Una estructura productiva más diversa se asocia a un ambiente propicio a nuevas inversiones, mayor innovación y transmisión de conocimientos, entre otros efectos positivos. A este respecto, el presente artículo contribuye empíricamente al estudio del fenómeno mediante un abordaje regional con datos actualizados para todas las provincias argentinas. En particular, se analiza la diversidad industrial tanto en términos estáticos como dinámicos: por un lado, se describe y cuantifica el grado de diversidad productiva regional en la Argentina y su evolución a lo largo del período 1996-2012, y por otro se examina la relación entre dicho fenómeno y algunos factores económicos regionales asociados.

En términos estáticos, las jurisdicciones más diversas del país son la Ciudad Autónoma de Buenos Aires y las provincias de Buenos Aires, Santa Fe, Córdoba, San Luis y Neuquén. Este resultado puede estar asociado con el mayor grado de urbanismo de estas regiones, en un entorno de mayores capacidades productivas, institucionales y organizativas que incentiven la radicación de un gran número de empresas de diversos sectores, o con la existencia de estímulos de política industrial específicos. En contraposición con este grupo, las provincias con una estructura industrial menos diversa son las de base agroalimentaria (Tucumán, La Pampa, Entre Ríos, Salta, Río Negro, Mendoza y Jujuy), donde la rama alimentos y bebidas concentra más de la mitad del empleo industrial.

En términos dinámicos, la diversidad productiva es un fenómeno procíclico, tanto a nivel nacional como en la mayor parte de las regiones. Este resultado (junto con el impacto negativo que sobre la diversidad tiene el cierre de empresas) pone de manifiesto la necesidad de contar con una senda de crecimiento estable para lograr un cambio estructural basado en la diversidad productiva. Al mismo tiempo, abre una serie de interrogantes acerca del impacto de las políticas macroeconómicas (cambiarias, de protección comercial y fiscales, entre otras) sobre la diversidad productiva, tanto a nivel nacional como regional.

Las provincias favorecidas con regímenes de promoción industrial (San Luis, Catamarca, San Juan, La Rioja y Tierra del Fuego) constituyen un caso particular en que la diversidad industrial no es procíclica sino que disminuye en forma continua. En algunos casos, esto puede estar asociado a la incapacidad actual de los regímenes de promover cambios en la estructura productiva que impliquen el crecimiento de ramas poco tradicionales, mientras que en otros la mayor especialización se relaciona con los grandes incentivos recibidos recientemente para el desarrollo de ciertas industrias, como la de aparatos de radio y televisión en Tierra del Fuego.

Los resultados de las estimaciones econométricas indican que la diversidad industrial de las provincias se asocia positivamente con el grado de desarrollo, el tamaño de la región y un mayor grado de urbanización. Esto implica una restricción al momento de fomentar la diversificación productiva a través de políticas específicas, dado que estas características estructurales son difíciles de modificar en el corto plazo. La diversidad industrial regional también se relaciona en forma directa con las capacidades territoriales, que podrían ser impulsadas a través de políticas industriales a mediano plazo.

Por otra parte, la salida de empresas se asocia negativamente con el grado de diversidad. Esto puede ser resultado del cierre de empresas en sectores poco tradicionales, cuyas economías externas (mercado de trabajo especializado y proveedores, entre otras) e instituciones de apoyo no están lo suficientemente consolidadas. Al respecto, las políticas de retención de empresas en sectores menos tradicionales pueden ser más efectivas para aumentar la diversidad que aquellas orientadas al ingreso de nuevos emprendimientos en dichos sectores.

Algunas dimensiones que no han sido abordadas en el presente artículo dan lugar a futuras investigaciones en la materia. En primer lugar, un mayor nivel de desagregación de los datos (tres o 
cuatro dígitos en la Clasificación Industrial Internacional Uniforme, Rev. 3.1), así como el estudio de los sectores primario, comercial y de servicios permitirían comprender con mayor profundidad el fenómeno, teniendo en cuenta que la importancia relativa de la industria difiere marcadamente entre provincias. Otro punto de interés consiste en determinar si la diversidad tiene lugar en industrias relacionadas o no, puesto que la literatura especializada internacional indica que estos dos tipos de diversidad son impulsados por diferentes fuentes y tienen distintos efectos. En la misma línea, la disponibilidad de datos de demografía empresarial con mayor apertura sectorial permitiría identificar en qué ramas de actividad (tradicionales o no) se producen efectivamente las entradas y salidas de empresas.

\section{Bibliografía}

Al-Marhubi, F. (2000), "Export diversification and growth: an empirical investigation", Applied Economics Letters, vol. 7, No 9, Abingdon, Taylor \& Francis.

Artopoulos, A., D. Friel y J. C. Hallak (2013), "Export emergence of differentiated goods from developing countries: export pioneers and business practices in Argentina", Journal of Development Economics, vol. 105, Amsterdam, Elsevier.

Aw, B. y G. Batra (1998), "Firm size and the pattern of diversification", International Journal of Industrial Organization, vol. 16, № 3, Amsterdam, Elsevier.

Baldwin, J. R. y otros (2000), "Patterns of corporate diversification in Canada: an empirical analysis", Research Paper, N ${ }^{\circ} 150$, Ottawa, Statistics Canada.

Baltagi, B. (2008), Econometric Analysis of Panel Data, West Sussex, John Wiley \& Sons.

Bebczuk, R. y D. Berrettoni (2006), "Explaining export diversification: an empirical analysis", Documentos de Trabajo, N 65, La Plata, Universidad Nacional de La Plata.

Beckstead, D. y W. M. Brown (2003), "From Labrador City to Toronto: the industrial diversity of Canadian cities, 1992 to 2002", Insights on the Canadian Economy, Ottawa, Ministerio de Industria.

Bielschowsky, R., M. Izam y N. Mulder (2011), "Dos estudios de la evolución del pensamiento de la CEPAL sobre la diversificación productiva y la inserción internacional (1949-2008)", serie Comercio Internacional, № 112 (LC/L.3415), Santiago, Comisión Económica para América Latina y el Caribe (CEPAL).

Bishop, P. y P. Gripaios (2007), "Explaining spatial patterns of industrial diversity: an analysis of sub-regions in Great Britain", Urban Studies, vol. 44, № 9, Thousand Oaks, Sage.

Boschma R. (2004), "Competitiveness of regions from an evolutionary perspective", Regional Studies, vol. 38, $N^{\circ}$ 9, Abingdon, Taylor \& Francis.

Boschma, R. y G. Capone (2015), "Institutions and diversification: related versus unrelated diversification in a varieties of capitalism framework", Research Policy, vol. 44, № 10, Amsterdam, Elsevier.

Boschma, R. y S. lammarino (2007), "Related variety and regional growth in Italy", SPRU Electronic Working Paper, N 162, Brighton, Universidad de Sussex.

Boschma, R., A. Minondo y M. Navarro (2013), "The emergence of new industries at the regional level in Spain: a proximity approach based on product relatedness", Economic Geography, vol. 89, № 1, Abingdon, Taylor \& Francis.

Cadot, O., C. Carrère y V. Strauss-Kahn (2011), "Export diversification: what's behind the hump?", Review of Economics and Statistics, vol. 93, № 2, Cambridge, MIT Press.

Capello, R. (2006), "La economía regional tras cincuenta años: desarrollos teóricos recientes y desafíos futuros", Investigaciones Regionales, № 9, Alcalá de Henares, Universidad de Alcalá.

Castellacci, F. (2007), "Technological regimes and sectoral differences in productivity growth", Industrial and Corporate Change, vol. 16, Nㅜ 6, Oxford, Oxford University Press.

Castillo, V. y otros (2004), "Observatorio de empleo y dinámica empresarial en Argentina", serie Desarrollo Productivo, № 148 (LC/L.2072-P), Comisión Económica para América Latina y el Caribe (CEPAL).

Crépon, B., E. Duguet y J. Mairesse (1998), "Research, innovation and productivity: an econometric analysis at the firm level", Economics of Innovation and New Technology, vol. 7, № 2, Abingdon, Taylor \& Francis.

De Benedictis, L., M. Gallegati y M. Tamberi (2009), "Overall trade specialization and economic development: countries diversify", Review of World Economics, vol. 145, No 1, Nueva York, Springer.

Dewhurst, J. H. L. y P. McCann (2002), "A comparison of measures of industrial specialization for travel-towork areas in Great Britain, 1981-1997”, Regional Studies, vol. 36, N5 5, Abingdon, Taylor \& Francis. 
Donato, V. (2007), "Políticas públicas y localización industrial en Argentina", Revista OIDLES, vol. 1, № 1, Málaga, Universidad de Málaga.

Dumais, G., G. Ellison y E. Glaeser (2002), "Geographic concentration as a dynamic process", The Review of Economics and Statistics, vol. 84, № 2, Cambridge, MIT Press.

Duranton, G. y D. Puga (2000), "Diversity and specialisation in cities: why, where and when does it matter?", Urban Studies, vol. 37, №3, Thousand Oaks, Sage.

Elhiraika, A. y M. Mbate (2014), "Assessing the determinants of export diversification in Africa", Applied Econometrics and International Development, vol. 14, № 1, Universidad de Santiago de Compostela.

Essletzbichler, J. y D. L. Rigby (2007), "Exploring evolutionary economic geographies”, Journal of Economic Geography, vol. 7, № 5, Oxford, Oxford University Press.

Filipescu, D. A. y otros (2013), "Technological innovation and exports: unpacking their reciprocal causality", Journal of International Marketing, vol. 21, № 1, Thousand Oaks, Sage.

Frenken, K., F. van Oort y T. Verburg (2007), "Related variety, unrelated variety and regional economic growth", Regional Studies, vol. 41, № 5, Abingdon, Taylor \& Francis.

Frenken, K. y otros (2004), "Variety and regional economic growth in the Netherlands", Papers in Evolutionary Economic Geography, № 0502, Utrecht, Universidad de Utrecht.

Fritzsche, F. J. y M. Vio (2000), "Especialización y diversificación industrial en la Región Metropolitana de Buenos Aires", EURE, vol. 26, № 79, Santiago, Pontificia Universidad Católica de Chile.

Fujita, M. y P. Krugman (2004), "La nueva geografía económica: pasado, presente y futuro", Investigaciones Regionales, № 4, Alcalá de Henares, Universidad de Alcalá.

Gatto, F. (2007), "Crecimiento económico y desigualdades territoriales: algunos límites estructurales para lograr una mayor equidad", Crisis, recuperación y nuevos dilemas: la economía argentina 2002-2007 (LCM.165-LC/BUE/W.20), B. Kosacoff (ed.), Santiago, Comisión Económica para América Latina y el Caribe (CEPAL).

Ghosh, A. y J. Ostry (1994), "Export instability and the external balance in developing countries", IMF Working Paper, № 94/8, Washington, D.C., Fondo Monetario Internacional (FMI).

Glaeser, E. L. y otros (1992), "Growth in cities", Journal of Political Economy, vol. 100, № 6, Chicago, Universidad de Chicago.

Guesnier, B. (1994), "Regional variations in new firm formation in France", Regional Studies, vol. 28, № 4, Abingdon, Taylor \& Francis.

Haddad, M., J. J. Lim y C. Saborowski (2010), "Trade openness reduces growth volatility when countries are well diversified”, World Bank Policy Research Working Paper, № WPS 5222, Washington, D.C., Banco Mundial.

Hammouda, H. y otros (2006), "Diversification: towards a new paradigm for Africa's development", ATPC Work in Progress, № 35, Adis Ababa, Comisión Económica para África.

Hausman, J. A. (1978), "Specification tests in econometrics", Econometrica, vol. 46, № 6, Cleveland, The Econometric Society.

$\mathrm{He}, \mathrm{X}$. (2009), "Corporate diversification and firm value: evidence from post-1997 data", International Review of Finance, vol. 9, № 4, Hoboken, Wiley.

Henderson, V., A. Kuncoro y M. Turner (1995), "Industrial development in cities", Journal of Political Economy, vol. 103, № 5, Chicago, The University of Chicago Press.

Herzer, D. y F. Nowak-Lehnmann (2006), "What does export diversification do for growth? An econometric analysis", Applied Economics, vol. 38, № 15, Abingdon, Taylor \& Francis.

Hesse, H. (2009), "Export diversification and economic growth", Breaking into New Markets: Emerging Lessons for Export Diversification, R. S. Newfarmer, W. Shaw y P. Walkenhorst (eds.), Washington, D.C., Banco Mundial.

Hidalgo, C. A. y R. Hausmann (2010), "Country diversification, product ubiquity, and economic divergence", CID Working Paper, N ${ }^{\circ} 201$, Cambridge, Centro para el Desarrollo Internacional, Harvard University.

Hidalgo, C. A. y otros (2007), "The product space conditions the development of nations", Science, vol. 317, $N^{\circ}$ 5837, Washington, D.C., Asociación Estadounidense para el Progreso de la Ciencia.

Hoover, Jr., E. M. (1936), "The measurement of industrial localization", The Review of Economics and Statistics, vol. 18, № 4, Cambridge, MIT Press.

Imbs, J. y R. Wacziarg (2003), "Stages of diversification", The American Economic Review, vol. 93, № 1, Nashville, Asociación Estadounidense de Economía.

Kamien, M. I. y N. L. Schwartz (1975), "Market structure and innovation: a survey", Journal of Economic Literature, vol. 13, № 1, Nashville, Asociación Estadounidense de Economía. 
Klinger, B. y D. Lederman (2004), "Discovery and development: an empirical exploration of 'new' products", Policy Research Working Paper, № 3450, Washington, D.C., Banco Mundial.

Kosacoff, B. y A. Ramos (1999), "El debate sobre política industrial", Revista CEPAL, № 68 (LC/G.2039-P), Santiago, Comisión Económica para América Latina y el Caribe (CEPAL).

Krugman, P. (1999), "The role of geography in development", International Regional Science Review, vol. 22, $N^{\circ}$ 2, Nueva York, Sage.

_ (1998), "What's new about the new economic geography?", Oxford Review of Economic Policy, vol. 14, № 2, Oxford, Oxford University Press.

(1995), Development, Geography and Economic Theory, Cambridge, MIT Press.

(1991), "Increasing returns and economic geography", Journal of Political Economy, vol. 99, №3, Chicago, Universidad de Chicago.

Lavarello, P. J. y M. Sarabia (2015), "La política industrial en la Argentina durante la década de 2000", serie Estudios y Perspectivas-Oficina de la CEPAL en Buenos Aires, № 45 (LC/L.414- LC/BUE/L.224), Santiago.

Lawson, C. (1999), "Towards a competence theory of the region", Cambridge Journal of Economics, vol. 23, $N^{\circ}$ 2, Oxford, Oxford University Press.

Mack, E., T. Grubesic y E. Kessler (2007), "Indices of industrial diversity and regional economic composition", Growth and Change, vol. 38, N $\mathrm{N}^{\circ}$, Hoboken, Wiley.

Marra, A., V. Carlei y A. L. Paglialunga (2011), "Economic diversity and industry patterns in Italian provinces: an empirical analysis", L'industria, vol. 32, № 3, Bolonia, Società editrice il Mulino.

Martin, R. y P. Sunley (2006), "Path dependence and regional economic evolution", Journal of Economic Geography, vol. 6, № 4, Oxford, Oxford University Press.

Mazorra, X. y A. Beccaria (2007), "Especialización productiva y empleo en Áreas Económicas Locales", Estructura productiva y empleo. Un enfoque transversal, M. Novick y H. Palomino (coords.), Buenos Aires, Ministerio de Trabajo, Empleo y Seguridad Social.

McCann, P. y F. van Oort (2009), "Theories of agglomeration and regional economic growth: a historical review", Handbook of Regional Growth and Development Theories, R. Capello y P. Nijkamp (eds.), Cheltenham, Edward Elgar.

Ministerio de Industria (2011), Plan Estratégico Industrial 2020, Buenos Aires.

Monastiriotis, V. (2000), "City size and production diversity: patterns of specialisation and diversity in the US cities, 1969-1997", 40 Congreso de la Asociación Europea de Ciencia Regional, Barcelona, 29 agosto- 1 septiembre.

Moncayo Jiménez, E. (2001), "Evolución de los paradigmas y modelos interpretativos del desarrollo territorial", serie Gestión Pública, N 13 (LC/L.1587-P-LC/IP/L.190), Santiago, Comisión Económica para América Latina y el Caribe (CEPAL).

Montgomery, C. A. (1994), "Corporate diversification", The Journal of Economic Perspectives, vol. 8, № 3, Nashville, Asociación Estadounidense de Economía.

Nachum, L. (2004), "Geographic and industrial diversification of developing country firms", Journal of Management Studies, vol. 41, № 2, Hoboken, Wiley.

Neffke, F., M. Henning y R. Boschma (2011), "How do regions diversify over time? Industry relatedness and the development of new growth paths in regions", Economic Geography, vol. 87, Nㅜ 3, Abingdon, Taylor \& Francis.

Neffke, F. y otros (2014), "Agents of structural change. The role of firms and entrepreneurs in regional diversification", Papers in Evolutionary Economic Geography, № 1410, Utrecht, Universidad de Utrecht.

Noseleit, F. (2010), "Market selection and regional diversification - empirical regularities from German paneldata", documento presentado en el $50^{\circ}$ Congreso de la Asociación Europea de Ciencia Regional, Jönköping, 19-23 agosto.

Parteka, A. y M. Tamberi (2011), "Export diversification and development - empirical assessment", Quaderni di Ricerca, №359, Ancona, Universita' Politecnica delle Marche.

Penrose, E. T. (1959), The Theory of the Growth of the Firm, Nueva York, Wiley.

Porta, F. (2016), "La estructura productiva argentina: problemas vigentes y desafíos pendientes", Voces en el Fénix, N 53, Buenos Aires, Universidad de Buenos Aires.

Ramanujan, V. y P. Varadarajan (1989), "Research on corporate diversification: a synthesis", Strategic Management Journal, vol. 10, № 6, Hoboken, Wiley.

Reynolds, P., D. Storey y P. Westhead (1994), "Cross-national comparisons of the variation in new firm formation rates", Regional Studies, vol. 28, № 4, Abingdon, Taylor \& Francis. 
Rodgers, A. (1957), "Some aspects of industrial diversification in the United States", Economic Geography, vol. 33, No 1, Abingdon, Taylor \& Francis.

Rojo Brizuela, S. y S. Rotondo (2006), Especialización industrial y empleo registrado en el Gran Buenos Aires, Buenos Aires, Ministerio de Trabajo, Empleo y Seguridad Social.

Schmookler, J. (1966), Invention and Economic Growth, Cambridge, Harvard University Press.

Teece, D. J. (1980), "Economies of scope and the scope of the enterprise", Journal of Economic Behavior \& Organization, vol. 1, № 3, Amsterdam, Elsevier.

Wooldridge, J. M. (2010), Econometric Analysis of Cross Section and Panel Data, Cambridge, MIT Press.

Zalduendo, E. (1975), "Las desigualdades económicas entre las regiones de Argentina" (CPRD-B/14), Santiago, Comisión Económica para América Latina y el Caribe (CEPAL). 


\section{Anexo A1}

Cuadro A1.1

Clasificación Industrial Internacional Uniforme (CIIU)

\begin{tabular}{|c|c|}
\hline Código & Rama de actividad \\
\hline 15 & Elaboración de productos alimenticios y bebidas \\
\hline 16 & Elaboración de productos de tabaco \\
\hline 17 & Fabricación de productos textiles \\
\hline 18 & Fabricación de prendas de vestir; adobo y teñido de pieles \\
\hline 19 & Curtido y adobo de cueros; fabricación de maletas, artículos de talabartería y calzado \\
\hline 20 & Producción de madera y fabricación de productos de madera y corcho, excepto muebles. \\
\hline 21 & Fabricación de papel y de productos de papel \\
\hline 22 & Actividades de edición e impresión y de reproducción de grabaciones \\
\hline 23 & Fabricación de coque, productos de la refinación del petróleo y combustible nuclear \\
\hline 24 & Fabricación de sustancias y productos químicos \\
\hline 25 & Fabricación de productos de caucho y plástico \\
\hline 26 & Fabricación de otros productos minerales no metálicos \\
\hline 27 & Fabricación de metales comunes \\
\hline 28 & Fabricación de productos elaborados de metal, excepto maquinaria y equipo \\
\hline 29 & Fabricación de maquinaria y equipo n.c.p. ${ }^{a}$ \\
\hline 30 & Fabricación de maquinaria de oficina, contabilidad e informática \\
\hline 31 & Fabricación de maquinaria y aparatos eléctricos n.c.p. ${ }^{\mathrm{a}}$ \\
\hline 32 & Fabricación de equipo y aparatos de radio, televisión y comunicaciones \\
\hline 33 & Fabricación de instrumentos médicos, ópticos y de precisión y fabricación de relojes \\
\hline 34 & Fabricación de vehículos automotores, remolques y semirremolques \\
\hline 35 & Fabricación de otros tipos de equipo de transporte \\
\hline 36 & Fabricación de muebles; industrias manufactureras n.c.p. ${ }^{a}$ \\
\hline 37 & Reciclado \\
\hline
\end{tabular}

Fuente: Elaboración propia, sobre la base de Naciones Unidas, "Clasificación Industrial Internacional Uniforme de todas las actividades económicas (CIIU). Revisión 3.1”, Informes Estadísticos, № 4, Nueva York, 2002.

a No clasificado previamente. 


\section{Anexo A2}

Cuadro A2.1

Argentina: matriz de correlación

\begin{tabular}{|c|c|c|c|c|c|c|c|c|c|c|}
\hline & $\mathrm{DP}^{\mathrm{a}}$ & $\begin{array}{c}\mathrm{Pbg}^{\mathrm{b}} \\
(\mathrm{IPI} \mathrm{BA})^{\mathrm{c}}\end{array}$ & $\begin{array}{c}\text { pbg_pc }{ }^{d} \\
(\mathrm{PII} B A)\end{array}$ & $\begin{array}{c}\mathrm{pbg} \\
(\mathrm{IPI} \text { Arg) }\end{array}$ & $\begin{array}{l}\text { pbg_pc } \\
\text { (IPI Arg) }\end{array}$ & Densidad & $\begin{array}{l}\text { Gasto } \\
\mathrm{I}+\mathrm{D}^{f}\end{array}$ & p_expo & Entrada & Salida \\
\hline DP & 1,00 & & & & & & & & & \\
\hline pbg (IPI BA) & 0,69 & 1,00 & & & & & & & & \\
\hline pbg_pc (IPI BA) & 0,40 & 0,34 & 1,00 & & & & & & & \\
\hline pbg (IPI Arg) & 0,69 & 1,00 & 0,34 & 1,00 & & & & & & \\
\hline pbg_pc (IPI Arg) & 0,40 & 0,34 & 1,00 & 0,34 & 1,00 & & & & & \\
\hline Densidad & $-0,07$ & $-0,12$ & 0,38 & $-0,12$ & 0,38 & 1,00 & & & & \\
\hline Gasto I+D & 0,49 & 0,72 & 0,26 & 0,72 & 0,25 & $-0,18$ & 1,00 & & & \\
\hline p_expo & 0,50 & 0,74 & 0,01 & 0,74 & 0,01 & $-0,10$ & 0,53 & 1,00 & & \\
\hline Entrada & 0,65 & 0,91 & 0,22 & 0,92 & 0,22 & $-0,12$ & 0,67 & 0,79 & 1,00 & \\
\hline Salida & 0,65 & 0,90 & 0,25 & 0,90 & 0,24 & $-0,12$ & 0,63 & 0,75 & 0,84 & 1,00 \\
\hline
\end{tabular}

Fuente: Elaboración propia.

a Diversidad productiva.

b Producto bruto geográfico.

c Índice de precios implícitos de Buenos Aires.

d Producto bruto geográfico per cápita.

e Índice de precios implícitos de la Argentina.

Gasto en investigación y desarrollo. 


\section{Anexo A3}

\section{Gráfico A3.1}

Argentina: evolución del índice de diversidad productiva por provincias

\section{A. Núcleo productivo y de consumo}

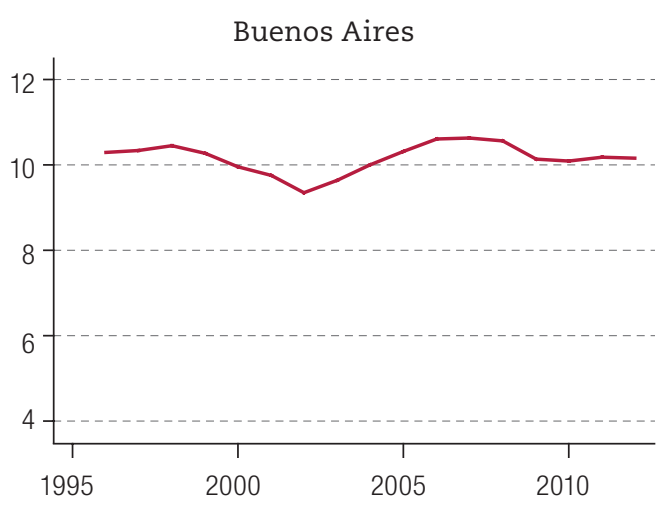

Ciudad Autónoma de Buenos Aires

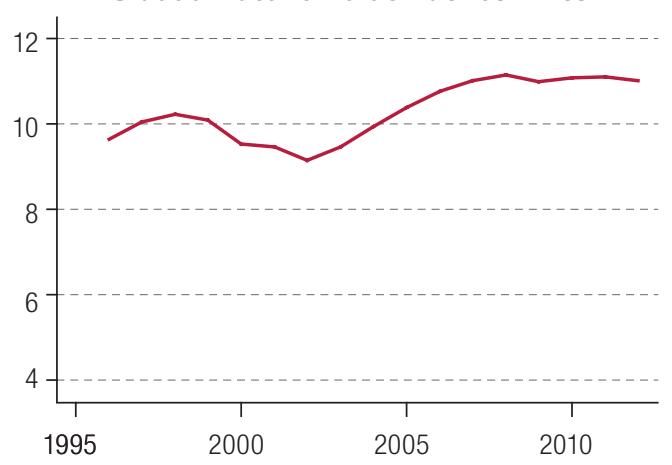

Córdoba
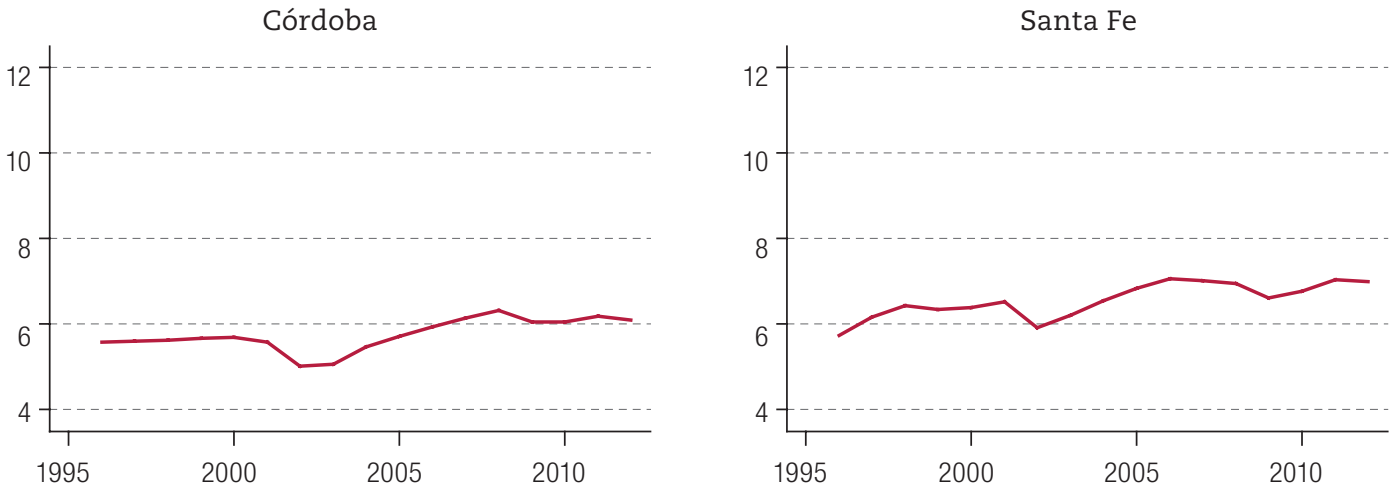

B. Con regímenes de promoción industrial
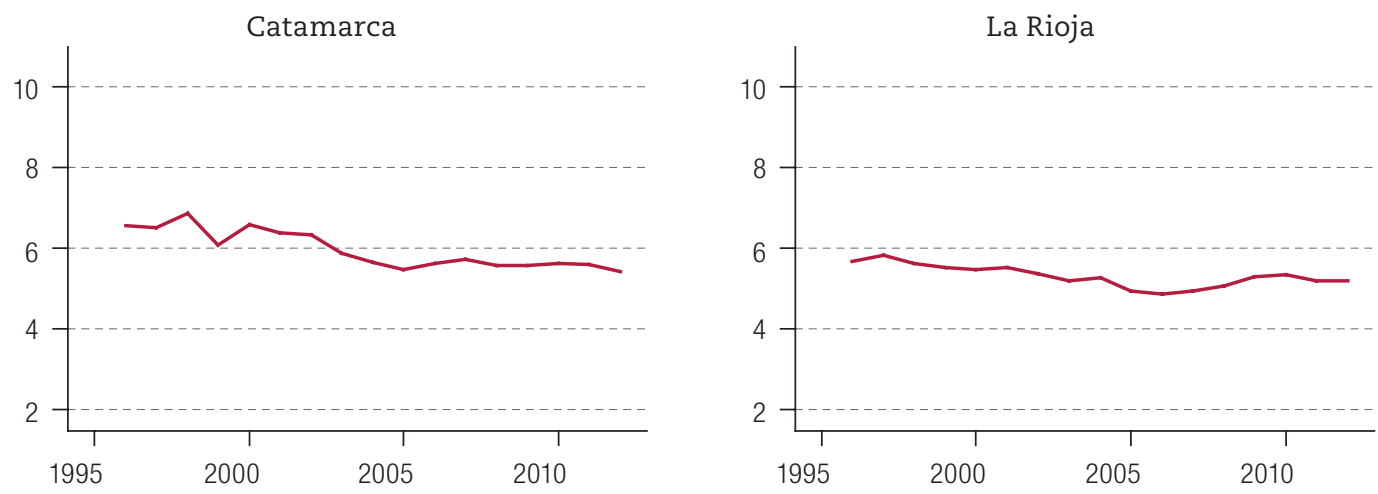
Gráfico A3.1 (continuación)
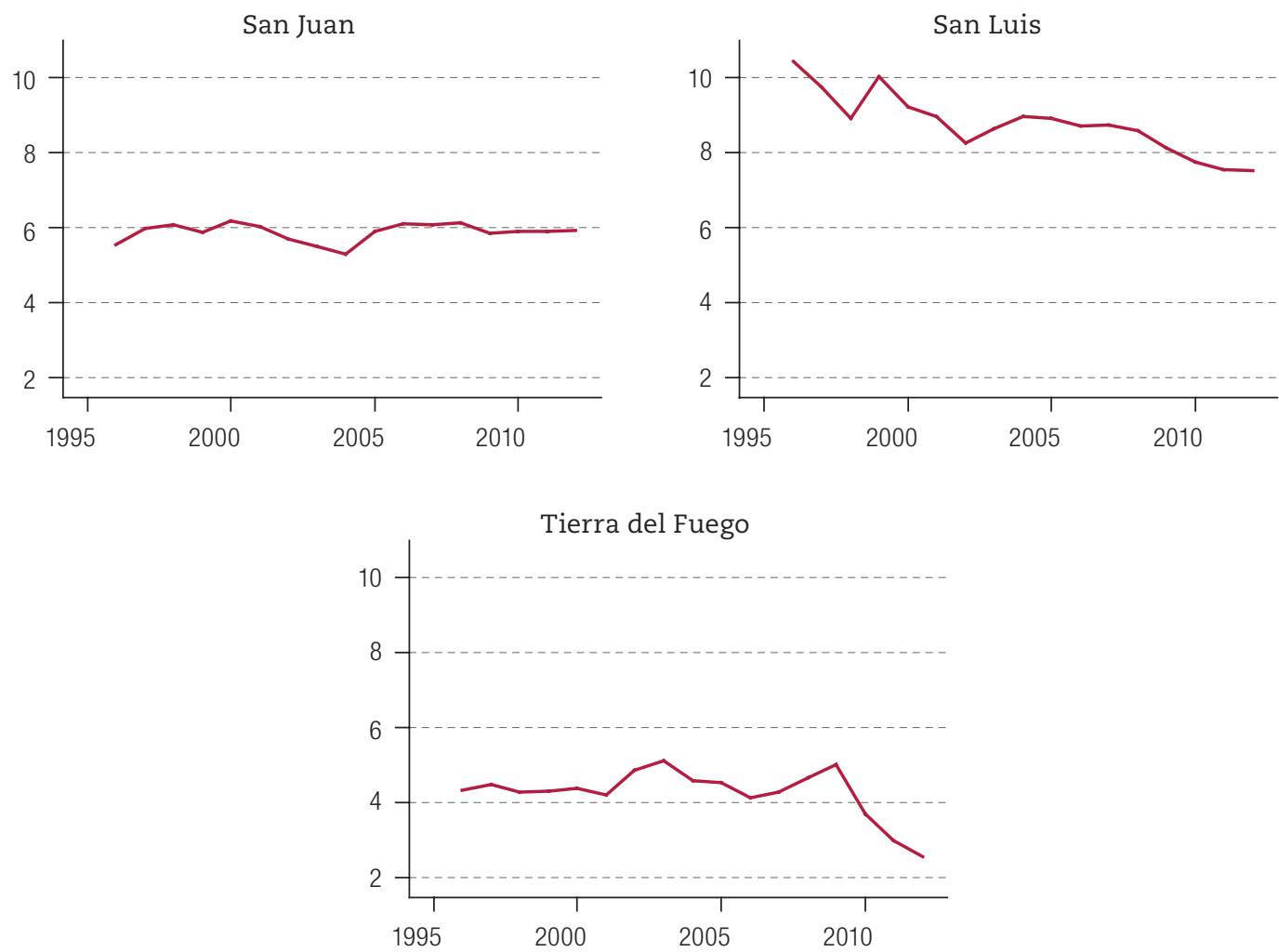

C. Complejo agroindustrial
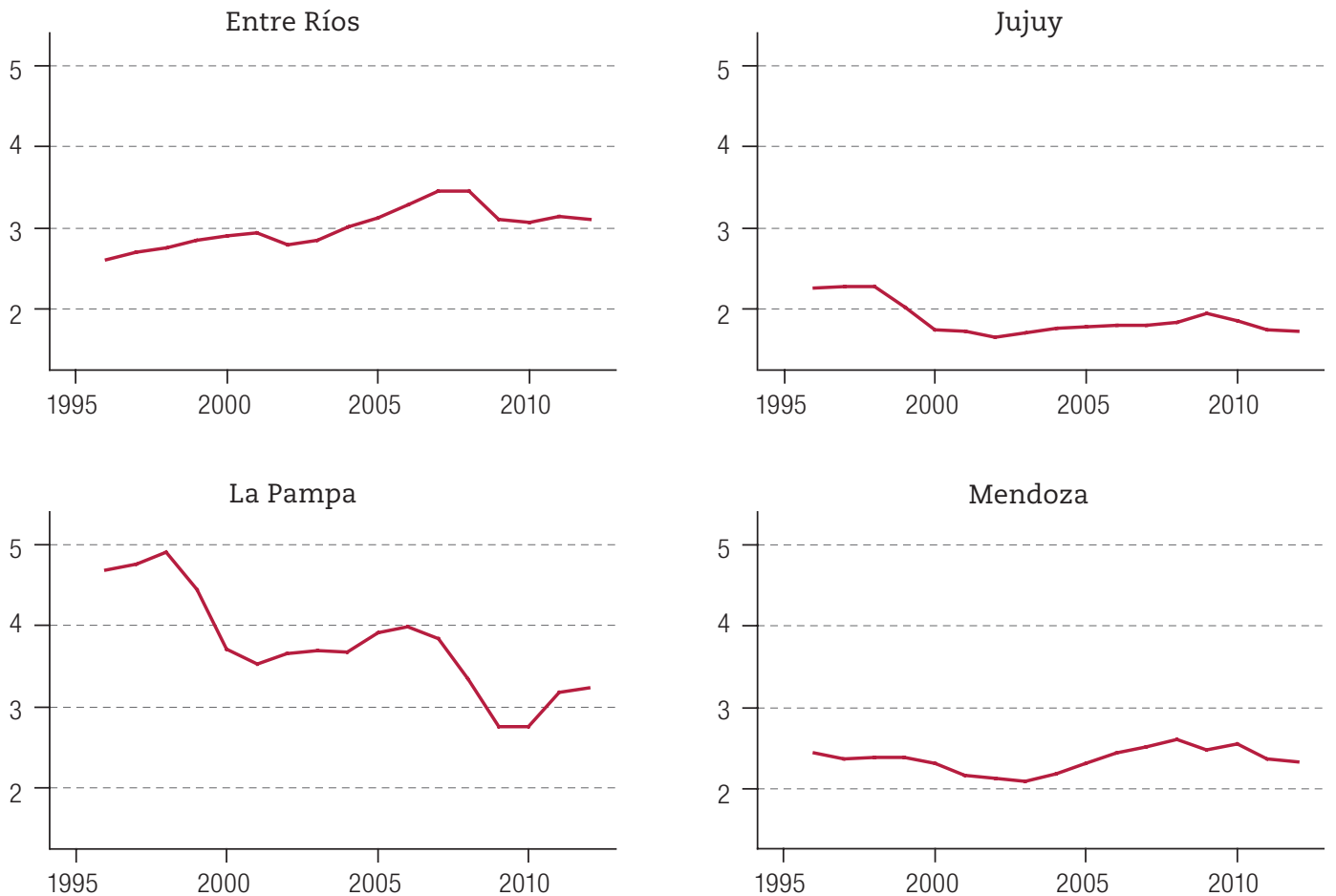
Gráfico A3.1 (continuación)
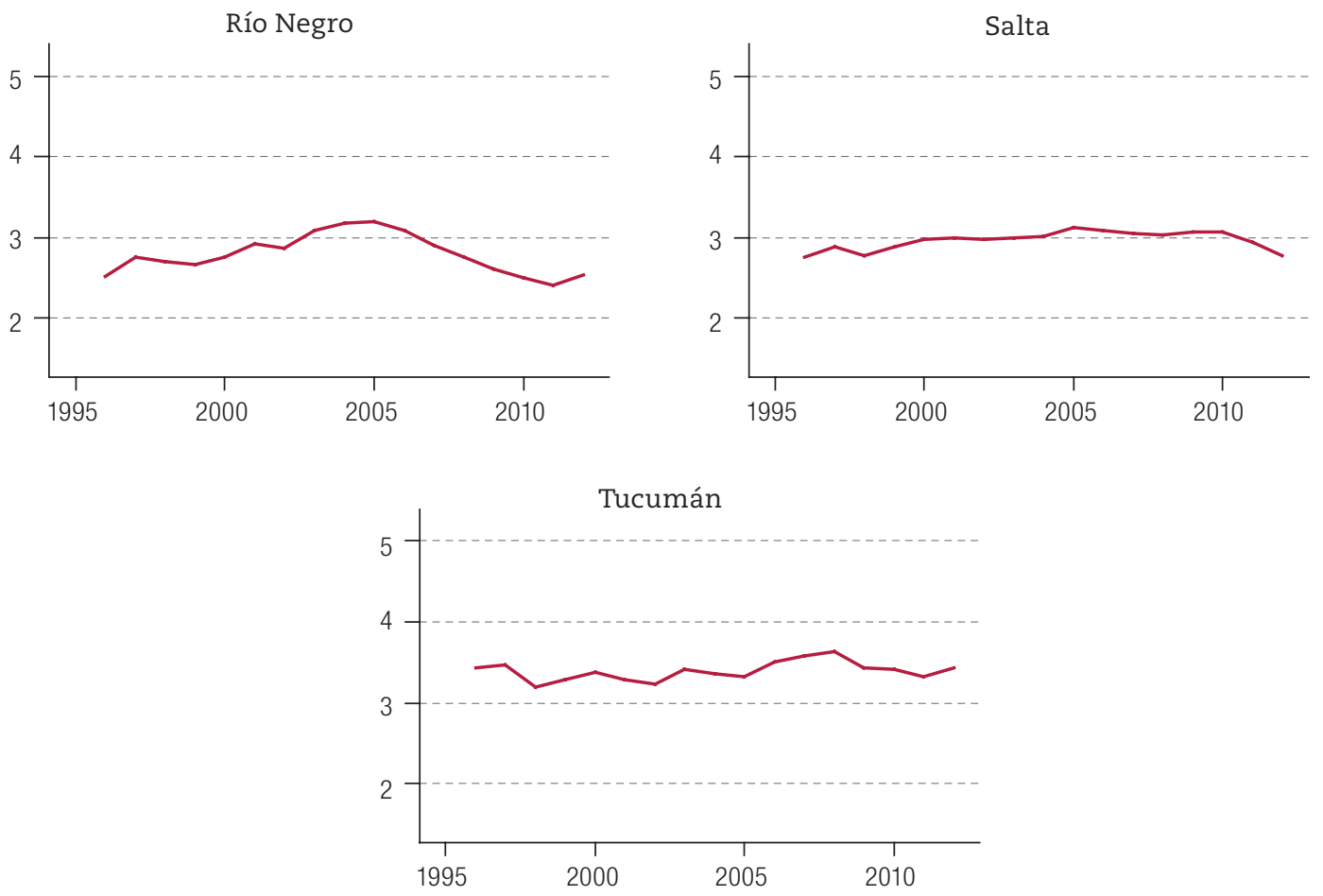

D. Complejos petrolero y pesquero
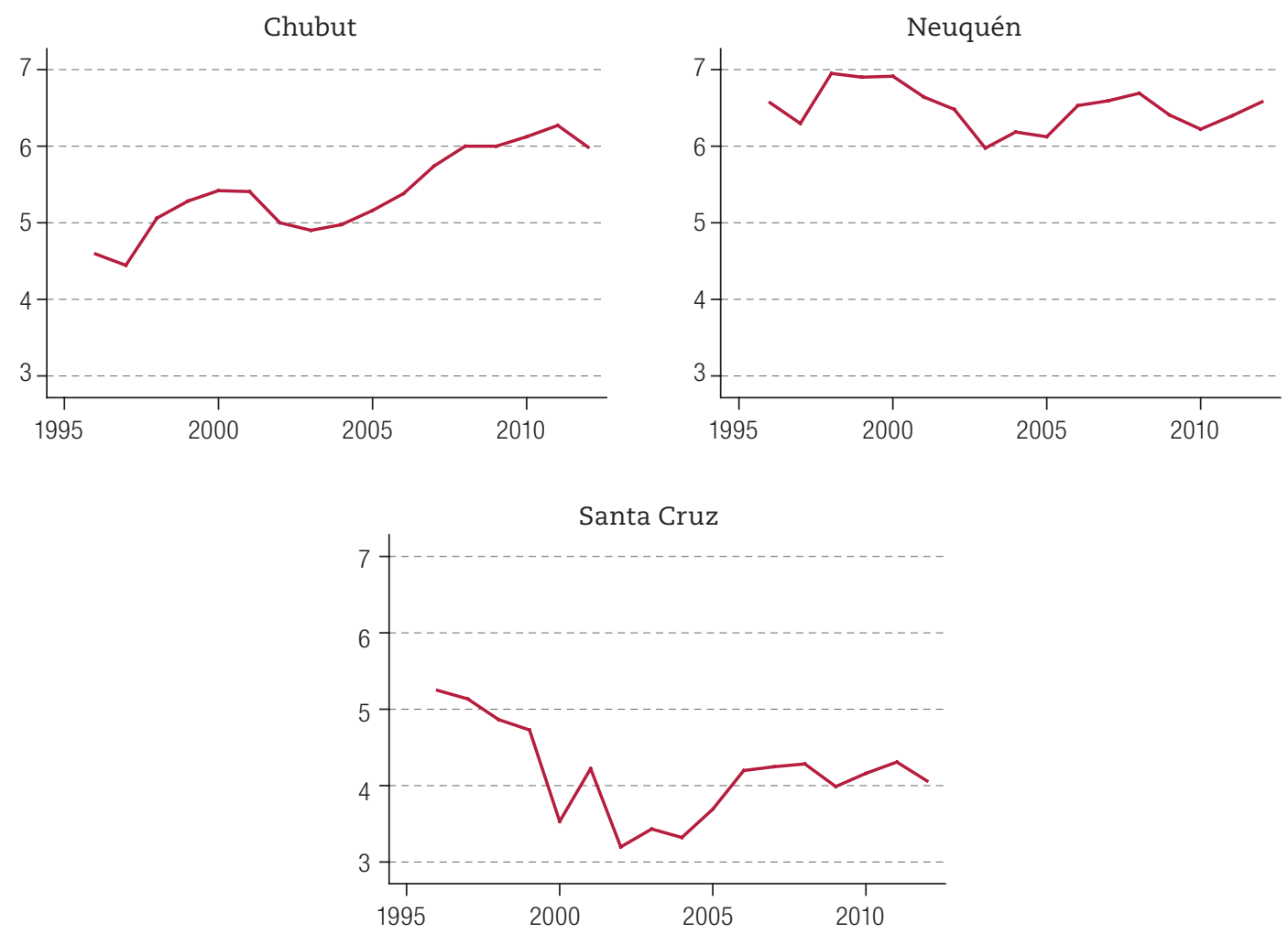
Gráfico A3.1 (conclusión)

E. Complejos forestal y textil
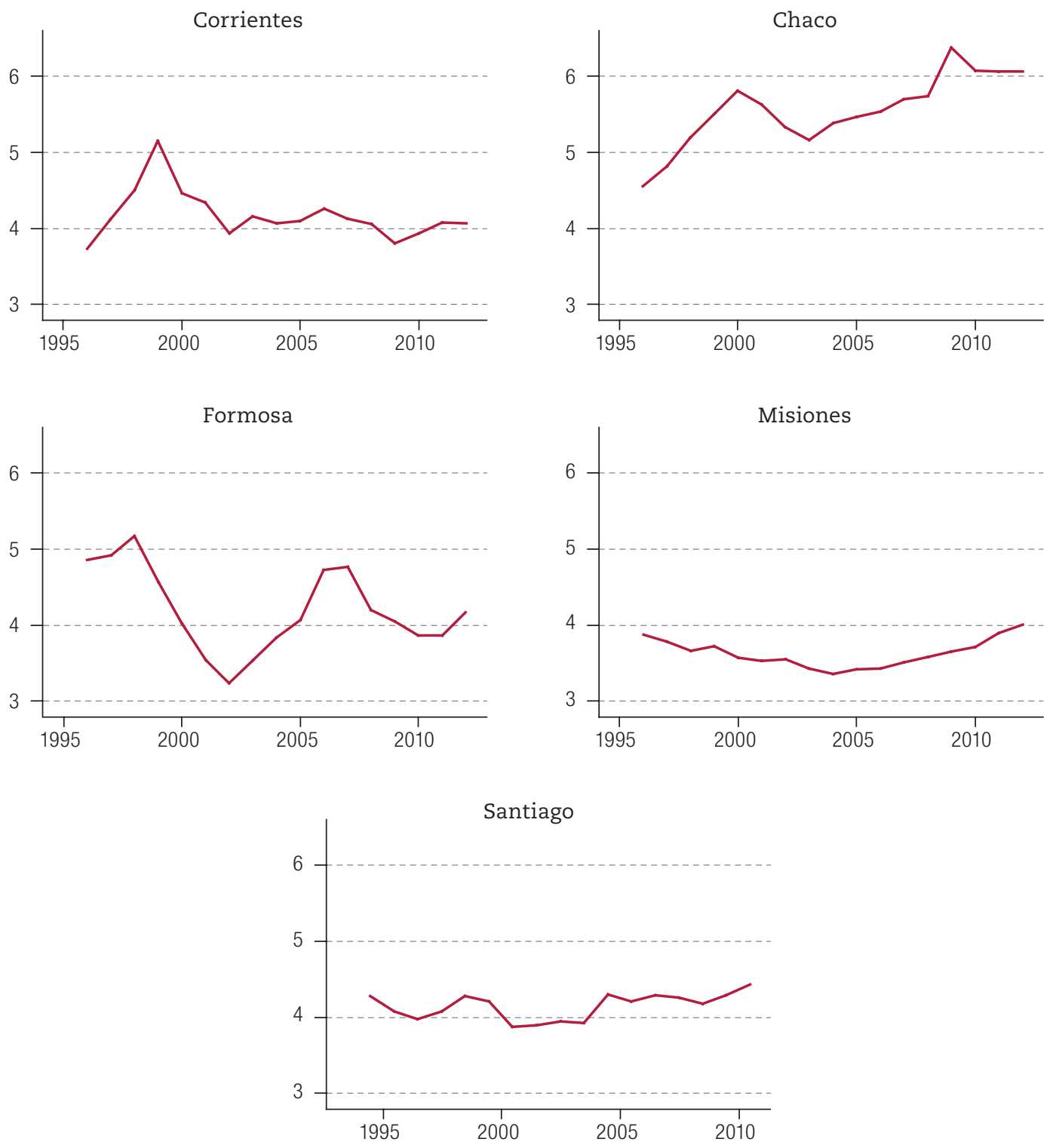

Fuente: Elaboración propia, sobre la base de la Base de Datos para el Análisis Dinámico del Empleo (BADE). 